\title{
EXTENDING HOLOMORPHIC SECTIONS FROM COMPLEX SUBVARIETIES
}

\author{
Franc Forstnerič and Jasna Prezelj
}

\section{\&1. Introduction and results.}

In this paper we treat the classical problem of extending holomorphic mappings and sections from closed complex subvarieties of a complex manifold. Our main results (theorems 1.1 and 1.4) extend those of Grauert [Gr2] and Cartan [Car]; results of this type are commonly referred to as the 'Oka-Grauert principle' on Stein manifolds. Our methods are similar to those developed in the papers of Gromov [Gro], Henkin and Leiterer [HL2] and the authors [FP1], [FP2]. The main addition here is the interpolation of a given holomorphic section on a complex subvariety of a Stein manifold. Our globalization scheme follows very closely the one developed in [FP2].

To state our first result we recall the notion of a (dominating) spray introduced by M. Gromov ([Gro], sec. 0.5). Given a holomorphic vector bundle $p: E \rightarrow Y$ over a complex manifold $Y$, we denote by $0_{y} \in E_{y}=p^{-1}(y)$ the zero element in the fiber $E_{y}$ and we observe that $E_{y}$ is a $\mathbf{C}$-linear subspace of the tangent space $T_{0_{y}} E$.

Definition 1. A spray on a complex manifold $Y$ is a holomorphic vector bundle $p: E \rightarrow$ $Y$, together with a holomorphic map $s: E \rightarrow Y$, such that for each $y \in Y, s\left(0_{y}\right)=y$ and the derivative $d s: T_{0_{y}} E \rightarrow T_{y} Y$ maps $E_{y}$ surjectively onto $T_{y} Y$.

1.1 Theorem. Let $X$ be a Stein manifold and $Y$ a complex manifold which admits a spray. Then for every closed complex subspace $X_{0} \subset X$ and every continuous map $f_{0}: X \rightarrow Y$ whose restriction to $X_{0}$ is holomorphic on $X_{0}$ there exists a holomorphic map $f_{1}: X \rightarrow Y$ such that $\left.f_{1}\right|_{X_{0}}=\left.f_{0}\right|_{X_{0}}$. Moreover, $f_{1}$ can be obtained from $f_{0}$ by a homotopy $f_{t}: X \rightarrow Y(t \in[0,1])$ which is fixed on $X_{0}$.

For maps of Stein manifolds into complex Lie groups or complex homogeneous spaces this was proved by Grauert ([Gra2], [Gra3]) and Cartan ([Car], Théorème 1 bis). The validity of theorem 1.1 (and of theorem 1.4 below) was asserted by Gromov ([Gro], sec. 2.9.C, p. 877), but very few details were provided there. The corresponding results with $X_{0}=\emptyset$ can be found in [Gro], and complete proofs are given in [FP1] and [FP2]. (For the general theory of Stein manifolds and Stein spaces we refer to [GuR] or [GRe].)

The best source of examples of complex manifolds with sprays is the following. Let $V_{1}, \ldots, V_{q}$ be $\mathbf{C}$-complete holomorphic vector fields on a complex manifold $Y$ which span the tangent space $T_{y} Y$ at each point $y \in Y$. Denote by $\theta_{t}^{j}$ the flow of $V_{j}$. Then the map $s: Y \times \mathbf{C}^{q} \rightarrow Y, s\left(y, t_{1}, \ldots, t_{q}\right)=\theta_{t_{1}}^{1} \circ \cdots \circ \theta_{t_{q}}^{q}(y)$ for $y \in Y$ and $\left(t_{1}, \ldots, t_{q}\right) \in \mathbf{C}^{q}$, is a spray on $Y$ (see [Gro] and [FP1]). Such sprays exist on complex Lie groups and homogeneous 
spaces (take left invariant holomorphic vector fields spanning the Lie algebra), and on spaces $Y=\mathbf{C}^{n} \backslash \Sigma$ where $\Sigma$ is an affine algebraic subvariety of codimension at least two (see $[\mathrm{FP} 2])$.

Theorem 1.1 reduces the holomorphic extension problem to a topological one. If the initial map $f_{0}$ is holomorphic in an open neighborhood of $X_{0}$ in $X$, the homotopy in theorem 1.1 can be chosen such that all sections $f_{t}$ are holomorphic in a neighborhood of $X_{0}$ (independent of $t$ ) and they agree with $f_{0}$ to a prescribed finite order along $X_{0}$ (see theorem 1.4). A local holomorphic extension of $f_{0}$ exists without any condition on $X$ and $Y$ provided that the subspace $X_{0}$ is Stein (proposition 1.3). Theorem 1.1 is a special case of theorem 1.4 below which gives extension results for sections of holomorphic submersions onto Stein manifolds.

We have already pointed out that theorem 1.1 applies to maps of Stein manifolds into $\mathbf{C}^{n} \backslash \Sigma$ where $\Sigma$ is an affine algebraic subvariety of codimension at least two. This does not hold for general analytic subvarieties, independently of their codimension.

Example 1. For each $n \geq 1$ there exists a discrete set $\Sigma \subset \mathbf{C}^{n}$ such that theorem 1.1 fails for maps of Stein manifolds into $Y=\mathbf{C}^{n} \backslash \Sigma$. (Note however that theorem 1.1 does hold when $\Sigma$ is a discrete set in $\mathbf{C}^{n}, n \geq 2$, which is tame in the sense of Rosay and Rudin $[\mathrm{RRu}]$, since the complement of such a set admits a spray.)

We obtain such examples as in the proof of theorem 1.6 (b) in [FP2]. Let $n=2$ for simplicity. By [RRu] there is a discrete set $\Sigma \subset \mathbf{C}^{2}$ such that the space $Y=\mathbf{C}^{2} \backslash \Sigma$ is volume hyperbolic, in the sense that any entire holomorphic map $g: \mathbf{C}^{n} \rightarrow Y$ has complex rank at most one at each point. The same is then true for holomorphic maps $f: X \rightarrow Y$ from any Stein manifold $X$ whose universal cover is a complex Euclidean space. We showed in [FP2] that consequently any such map is homotopic to a constant map $X \rightarrow$ point.

The set $X=\left(\mathbf{C}^{*}\right)^{2}=\mathbf{C}^{2} \backslash\{z w=0\}$ is a Stein manifold which is covered by $\mathbf{C}^{2}$. Choose a complex line $\Lambda \subset \mathbf{C}^{2}$ that intersects the set $\{z w=0\} \subset \mathbf{C}^{2}$ in precisely two points, say $p=(1,0)$ and $q=(0,1)$, and take $X_{0}=\Lambda \cap\left(\mathbf{C}^{*}\right)^{2}=\Lambda \backslash\{p, q\}$; this twice punctured complex line is a closed complex submanifold of $X$. We may choose the discrete set $\Sigma \subset \mathbf{C}^{2}$ as above such that $\Sigma \cap \Lambda=\{p, q\}$. Let $f_{0}: X_{0} \hookrightarrow Y=\mathbf{C}^{2} \backslash \Sigma$ be the inclusion map. By the choice of $\Sigma$ there is an open tube $U \subset \mathbf{C}^{2}$ around $\Lambda$ such that $U \cap \Sigma=\{p, q\}$. Clearly we can extend $f_{0}$ to a smooth map $\tilde{f}: X \rightarrow U \backslash\{p, q\} \subset Y$. However, $f_{0}$ has no holomorphic extension $f: X \rightarrow Y$ which is seen as follows. Any such extension would have rank at most one by the choice of $\Sigma$. Since $\left.f_{0}\right|_{X_{0}}$ already has rank one, it would follow that $f(X)=f_{0}\left(X_{0}\right)=X_{0}$. But $X_{0}$ is a twice punctured complex line and thus a hyperbolic space while $X$ is covered by $\mathbf{C}^{2}$; hence $f$ (and therefore $f_{0}$ ) would be constant, a contradiction.

When the manifold $Y$ is Stein, the condition in theorem 1.1 is almost necessary:

1.2 Proposition. (Gromov [Gro], 3.2.A.) Let $Y$ be a Stein manifold. Assume that for any Stein manifold $X$, any closed complex submanifold $X_{0} \subset X$ and any continuous map $f_{0}: X \rightarrow Y$ which is holomorphic in a neighborhood of $X_{0}$ there exists a holomorphic map $f: X \rightarrow Y$ which agrees with $f_{0}$ to the second order along $X_{0}$. Then $Y$ admits a spray.

Our proof in sect. 6 is slightly different from Gromov's proof. 
We now turn our attention to the extension problem for sections of holomorphic submersions. Let $h: Z \rightarrow X$ be a holomorphic submersion of a complex manifold $Z$ onto a complex manifold $X$. This means that that for each point $z \in Z$ the derivative $d_{z} h$ maps $T_{z} Z$ surjectively onto $T_{x} X, x=h(z)$. Let $Z_{x}=h^{-1}(x)$ for $x \in X$. We denote by $V T(Z)$ the kernel of $d h$ and call it the vertical tangent bundle (with respect to $h$ ). Clearly $V T(Z)$ is holomorphic subbundle of $T Z$ whose fiber at $z \in Z$ equals

$$
V T_{z}(Z)=\left\{v \in T_{z} Z: d_{z} h(v)=0\right\}=T_{z} Z_{h(z)}
$$

A submersion $h: Z \rightarrow X$ is locally trivial if each point $x \in X$ has an open neighborhood $U \subset X$ such that $h^{-1}(U)$ is equivalent to a product $U \times Y$ by a fiber preserving biholomorphic map $\Phi: h^{-1}(U) \rightarrow U \times Y$; in such case the submersion is a holomorphic fiber bundle over each connected component of $X$.

A section of $h: Z \rightarrow X$ over a subset $U \subset X$ is a continuous map $f: U \rightarrow Z$ such that $h(f(x))=x$ for all $x \in U$. If $U$ is an open subset of $X$ and $X_{0}$ is an analytic subset of $X$, we say that a section $f$ is holomorphic on $X_{0} \cup U$ if $\left.f\right|_{U}$ is holomorphic in $U$ and $\left.f\right|_{X_{0}}$ is holomorphic on $X_{0}$ (in the induced complex structure on $X_{0}$ ).

We first give a local extension theorem for sections of submersions.

1.3 Proposition. Let $h: Z \rightarrow X$ be a holomorphic submersion of a complex manifold $Z$ onto a complex manifold $X$. Given a closed Stein subspace $X_{0}$ of $X$ (possibly with singularities) and a holomorphic section $f_{0}:\left.X_{0} \rightarrow Z\right|_{X_{0}}$ of $h$ over $X_{0}$, there are an open set $U \subset X$ containing $X_{0}$ and a holomorphic section $f:\left.U \rightarrow Z\right|_{U}$ with $\left.f\right|_{X_{0}}=f_{0}$. If $f_{0}$ extends to a continuous section $f_{0}: X \rightarrow Z$, there is a homotopy $f_{t}: X \rightarrow Z(t \in[0,1])$ which is fixed on $X_{0}$ such that the section $f_{1}$ is holomorphic in an open set containing $X_{0}$.

Our proof of prop. 1.3 in sect. 6 applies also in the case when $X_{0}$ is a Stein subspace of a complex space $X$, possibly with singularities. The extension problem for maps $f: X_{0} \rightarrow Y$ can be reduced to that of sections of the trivial submersion $Z=X \times Y \rightarrow X$, and hence proposition 1.3 implies the existence of local holomorphic extensions in theorem 1.1. Proposition 1.3 remains valid for families of holomorphic sections depending continuously on a parameter in a compact Hausdorff space $P$ (compare def. 3 below).

We now consider the global extension problem for sections of submersions. If $p: E \rightarrow Z$ is a holomorphic vector bundle and $z \in Z$, we write $E_{z}=p^{-1}(z) \subset E$ and denote by $0_{z} \in E_{z}$ the zero element of $E_{z}$.

Definition 2. (Gromov [Gro], sec. 1.1.B.) Let $h: Z \rightarrow X$ be a holomorphic submersion and $U \subset X$ an open subset. A spray on $\left.Z\right|_{U}=h^{-1}(U)$ associated to $h$ (a fiber-dominating spray in Gromov's terminology) is a triple $(E, p, s)$, where $p:\left.E \rightarrow Z\right|_{U}$ is a holomorphic vector bundle and $s:\left.E \rightarrow Z\right|_{U}$ is a holomorphic map satisfying for each $\left.z \in Z\right|_{U}$

(i) $s\left(E_{z}\right) \subset Z_{h(z)}$ (equivalently, $\left.h \circ p=h \circ s\right)$,

(ii) $s\left(0_{z}\right)=z$, and

(iii) the restriction of the derivative $d s: T_{0_{z}} E \rightarrow V T_{z}(Z)$ to the subspace $E_{z} \subset T_{0_{z}} E$ maps $E_{z}$ surjectively onto $V T_{z}(Z)$. 
Thus a spray on a complex manifold $Y$ in the sense of definition 1 coincides with a fiber-spray associated to the trivial submersion of $Y$ to a point. We give examples of submersions with sprays after corollary 1.5 below.

We will have to consider parametrized families of sections and we now introduce the relevant notions; the reader may observe a close similarity with the objects that were considered by Grauert [Gr2] and Cartan [Car].

Definition 3. Let $P$ be nonempty compact Hausdorff spaces and $P_{0} \subset P$ a closed subset (possibly empty) which is a strong deformation retraction of an open neighborhood $U \supset P_{0}$ in $P$. (In our constructions $P$ will be a polyhedron and $P_{0} \subset P$ a subpolyhedron.)

(a) A P-section of a submersion $h: Z \rightarrow X$ is a continuous map $f: X \times P \rightarrow Z$ such that $f_{p}=f(\cdot, p): X \rightarrow Z$ is a section of $h$ for each fixed $p \in P$. A P-section $f$ is holomorphic on $X$ (resp. on a subset $X_{0} \subset X$ ) if $f_{p}$ is holomorphic on $X$ (resp. on $\left.X_{0}\right)$ for each fixed $p \in P$.

(b) A homotopy of $P$-sections is a $P \times[0,1]$-section, i.e., a continuous map $H: X \times P \times$ $[0,1] \rightarrow Z$ such that $H_{t}=H(\cdot, \cdot, t): X \times P \rightarrow Z$ is a $P$-section for each $t \in[0,1]$. The homotopy $H$ is holomorphic if the section $H_{p, t}=H(\cdot, p, t): X \rightarrow Z$ is holomorphic for each $(p, t) \in P \times[0,1]$.

(c) $A\left(P, P_{0}\right)$-section of $h$ is a $P$-section $f: X \times P \rightarrow Z$ such that the section $f_{p}=$ $f(\cdot, p): X \rightarrow Z$ is holomorphic on $X$ for each $p \in P_{0}$.

The following is the main result in this paper.

1.4 Theorem. Let $h: Z \rightarrow X$ be a holomorphic submersion of a complex manifold $Z$ onto a Stein manifold $X$. Let $X_{0} \subset X$ be a closed complex subvariety of $X, K \subset \subset X$ a compact $\mathcal{H}(X)$-convex subset and $U \subset X$ an open set containing $K$. Assume that for each point $x \in X \backslash K$ there is an open neighborhood $U_{x} \subset X$ such that $h: h^{-1}\left(U_{x}\right) \rightarrow U_{x}$ admits a spray (def. 2). Let $P$ be a compact Hausdorff space. For any $P$-section $f_{0}: X \times P \rightarrow Z$ of $h$ which is holomorphic on $X_{0} \cup U$ there are an open set $U^{\prime} \subset X$, with $K \subset U^{\prime} \subset U$, and a homotopy of $P$-sections $F: X \times P \times[0,1] \rightarrow Z$ such that, writing $f_{t}=F(\cdot, \cdot, t): X \times P \rightarrow Z$ for $t \in[0,1]$, we have

(i) $f_{0}$ is the given initial P-section,

(ii) the $P$-section $f_{1}$ is holomorphic on $X$,

(iii) For each $t \in[0,1]$ the $P$-section $f_{t}$ is holomorphic on $X_{0} \cup U^{\prime},\left.f_{t}\right|_{X_{0}}=\left.f_{0}\right|_{X_{0}}$, and $f_{t}$ approximates $f_{0}$ uniformly on $K$.

Moreover, if $f_{0}$ is a $\left(P, P_{0}\right)$-section, we can we can choose the homotopy $F$ as above such that it is fixed on $P_{0}$, i.e., the section $f_{p, t}$ is independent of $t \in[0,1]$ when $p \in P_{0}$.

If the initial $P$-section $f_{0}$ is holomorphic in an open set $V \supset X_{0} \cup K$, then for any integer $k \in \mathbf{Z}_{+}$we can choose $F$ as above which is in addition holomorphic in a neighborhood of $X_{0} \cup K$ and such that for each $(p, t) \in P \times[0,1]$, the section $f_{p, t}$ matches $f_{p, 0}$ to order $k$ along $X_{0}$. (In this case it suffices to assume that the submersion $h$ admits a spray in a small neighborhood of each point $x \in X \backslash\left(X_{0} \cup K\right)$, but we do not need a spray over points in $X_{0}$.) 
It is possible to extend theorem 1.4 to submersions with stratified sprays over Stein spaces (section 7). A special case of theorem 1.4 was proved in [FP2] (theorems 1.7 and 1.9 ), but the proof given there does not carry over to the present situation. Theorem 1.4 implies the following (see the proof of corollary 1.5 in [FP1]):

1.5 Corollary. Let $h: Z \rightarrow X$ be a holomorphic submersion, $X_{0} \subset X$ a closed complex subvariety and $f_{0}: X_{0} \rightarrow Z$ a holomorphic section of $h$ over $X_{0}$. Denote by $\mathcal{H}\left(X, Z ; X_{0}, f_{0}\right)$ (resp. $\mathcal{C}\left(X, Z ; X_{0}, f_{0}\right)$ the set of global holomorphic (resp. continuous) sections of $h: Z \rightarrow X$ whose restriction to $X_{0}$ equals $f_{0}$. (Both spaces are endowed with the compact-open topology.) Then the inclusion $\mathcal{H}\left(X, Z ; X_{0}, f_{0}\right) \hookrightarrow \mathcal{C}\left(X, Z ; X_{0}, f_{0}\right)$ is a weak homotopy equivalence, i.e., it induces an isomorphism of all homotopy groups of the two spaces. The same is true for the inclusion $\mathcal{H}(X, Z) \hookrightarrow \mathcal{C}(X, Z) \cap \mathcal{H}\left(X_{0}, Z\right)$ of the space of holomorphic sections into the space of continuous sections whose restrictions to $X_{0}$ are holomorphic.

Example 2. Fix an integer $q \geq 2$ and set

$$
\Gamma=\left\{\left(z^{\prime}, z_{q}\right) \in \mathbf{C}^{q}:\left|z_{q}\right| \leq 1+\left|z^{\prime}\right|\right\} .
$$

Let $h: V \rightarrow X$ be a holomorphic fiber bundle with fiber $\mathbf{C}^{q}$ and structure group Aut $\mathbf{C}^{q}$. Let $\Sigma \subset V$ be a closed complex subvariety in $V$ satisfying:

(a) $\Sigma_{x}=\Sigma \cap h^{-1}(x)$ is of complex dimension at most $q-2$ for each $x \in X$, and

(b) each point $x \in X$ has an open neighborhood $U \subset X$ and a fiber preserving biholomorphic map $\Phi: U^{\prime}=h^{-1}(U) \rightarrow U \times \mathbf{C}^{q}$ such that $\Phi\left(U^{\prime} \cap \Sigma\right) \subset U \times \Gamma$.

Then the submersion $h: Z=V \backslash \Sigma \rightarrow X$ admits a spray over each set $U$ as in (b) ([FP2], lemma 7.1) and hence theorem 1.4 applies. (For holomorphic vector bundles $V \rightarrow X$ this was proved in [FP2], theorem 1.7.) The total space $V$ of such a fiber bundle need not be Stein even when $X=\mathbf{C}$ and $q=2[\mathrm{Dem}]$.

Problem. For which compact sets $K \subset \mathbf{C}^{n}$ does the homotopy principle hold for maps of Stein manifolds into $\mathbf{C}^{n} \backslash K$, in the sense that each continuous map $f_{0}: X \rightarrow \mathbf{C}^{n} \backslash K$ is homotopic to a holomorphic map $f_{1}: X \rightarrow \mathbf{C}^{n} \backslash K$ ?

At this point the only known examples are the finite sets. Using analytic continuation it is easily seen that, unless $K$ is finite, there is no spray on $Y=\mathbf{C}^{n} \backslash K$ defined on a trivial bundle over $Y$ (but we don't know whether a spray may exist on a more general holomorphic vector bundles over $Y$ ). For instance, does the above h-principle hold when $K$ is a closed ball in $\mathbf{C}^{n}$ ? If so, this would give many holomorphic maps $f: X \rightarrow \mathbf{C}^{n}$ with $\inf _{x \in X}|f(x)|>0$, and such estimates are useful in the embedding-interpolation problems as is clear from [Pre]. A good test case might be the holomorphic map

$$
\mathrm{SL}(2, \mathbf{C}) \rightarrow \mathbf{C}^{2} \backslash\{0\}, \quad\left(\begin{array}{cc}
\alpha & \beta \\
\gamma & \delta
\end{array}\right) \rightarrow(\alpha, \beta) \quad(\alpha \delta-\beta \gamma=1)
$$

This map is homotopic to a smooth map into the complement of $B=\left\{z \in \mathbf{C}^{n}:|z| \leq 1\right\}$, but it is not clear whether it is homotopic to a holomorphic map to $\mathbf{C}^{n} \backslash B$. 
We prove propositions 1.2, 1.3 and theorem 1.4 in section 6 below. In the proof of theorem 1.4 we need the tools developed in sect. $2-5$. Theorem 1.1 is a special case of theorem 1.4 and will not be treated separately because we do not know any substantially simpler proof for this special case. In section 7 we discuss an extension of theorem 1.4 to submersions with stratified sprays over Stein spaces.

The basic analytic constructions in the proof of theorem 1.4 are similar to those in [Gra2], [Car], [Gro], [HL2], [FP1] and [FP2]. We first show how to patch holomorphic sections $a$ resp. $b$, defined on sets $A \subset X$ resp. $B \subset X$ and extending a given holomorphic section $f_{0}: X_{0} \rightarrow Z$ on a subvariety $X_{0} \subset X$, into a single holomorphic section over $\tilde{a}$ on $A \cup B$ such that $\left.\tilde{a}\right|_{A}$ approximates $a$ and $\tilde{a}=f_{0}$ on $(A \cup B) \cap X_{0}$. This can be done if $a$ and $b$ are sufficiently close on $A \cap B$ and if the sets $A$ and $B$ satisfy certain conditions (section 5). In the globalization scheme the basic analytic constructions are needed for parametrized families of sections, and for this reason we do everything by smooth Banach space operators. We globalize the construction using the scheme outlined in [Gro] and developed in [FP2]. We also need certain special coverings of the base Stein manifold constructed by Henkin and Leiterer in [HL2].

\section{\&2. Oka-Weil theorems for submersions with sprays.}

In this section we prove a homotopy version of the Oka-Weil approximation theorem for sections of submersions with sprays over Stein manifolds, with interpolation on a complex subvariety $X_{0}$ of $X$. This is similar to results in [Gr1], [Gro] and [FP1]. We first consider the case without parameters; theorem 2.1 coincides with theorem 4.1 in [FP1] when $X_{0}=\emptyset$.

2.1 Theorem. Let $h: Z \rightarrow X$ be a holomorphic submersion of a complex manifold $Z$ onto a Stein manifold $X$, let $X_{0} \subset X$ be a closed complex subvariety of $X$, and let $K$ be a compact $\mathcal{H}(X)$-convex subset. Assume that $U \supset K$ is an open set and $f_{t}: U \rightarrow Z$ $(t \in[0,1])$ is a homotopy of holomorphic sections of $h$ over $U$ such that $f_{0}$ extends to a holomorphic section over $X$ and the homotopy is fixed over $X_{0}$, i.e., $f_{t}(x)=f_{0}(x)$ for all $x \in X_{0}$ and $t \in[0,1]$. Let $d$ be a metric on $Z$ compatible with the manifold topology. If the submersion $h$ admits a spray (def. 2) then for each $\epsilon>0$ there exists a continuous family of holomorphic sections $\tilde{f}_{t}: X \rightarrow Z(t \in[0,1])$ such that

(a) $\tilde{f}_{0}=f_{0}$,

(b) $\left.\tilde{f}_{t}\right|_{X_{0}}=\left.f_{0}\right|_{X_{0}}$ for each $t \in[0,1]$, and

(c) $d\left(\tilde{f}_{t}(x), f_{t}(x)\right)<\epsilon$ for each $x \in K$ and $t \in[0,1]$.

Proof. Write $\tilde{X}=f_{0}(X) \subset Z$ and $\widetilde{X}_{0}=f_{0}\left(X_{0}\right) \subset \tilde{X}$. The restriction of the spray map $s: E \rightarrow Z$ to the holomorphic vector bundle $\widetilde{E}=\left.E\right|_{\widetilde{X}} \rightarrow \widetilde{X}$ is a submersion from an open neighborhood of the zero section in $\widetilde{E}$ onto an open neighborhood of $\widetilde{X}$ in $Z$. Hence there is a $t_{1}>0$ such that, after shrinking $U$ slightly around $K$, we can pull back the holomorphic sections $f_{t}: U \rightarrow Z$ for $0 \leq t \leq t_{1}$ to a continuous family of holomorphic sections $\xi_{t}: f_{0}(U) \rightarrow \widetilde{E}$ such that $\xi_{0}$ is the zero section, and such that $\xi_{t}$ vanishes on $f_{0}\left(U \cap X_{0}\right)$ for each $t \in\left[0, t_{1}\right]$. 
We may assume that $\bar{U}$ is compact. By the Oka-Cartan theory there exist finitely many holomorphic functions $g_{1}, \ldots, g_{k}: X \rightarrow \mathbf{C}$ which vanish on $X_{0}$ and which generate the ideal of $X_{0}$ at each point $x \in \bar{U}$. Since $\xi_{t} \circ f_{0}$ vanishes on $X_{0} \cap U$, we have

$$
\xi_{t}\left(f_{0}(x)\right)=\sum_{j=1}^{k} g_{j}(x) \xi_{t}^{j}\left(f_{0}(x)\right) \quad(x \in U)
$$

for some holomorphic sections $\xi_{t}^{j}: f_{0}(U) \rightarrow \widetilde{E}$ depending continuously on $t \in\left[0, t_{1}\right]$. We now apply the Oka-Weil theorem [Hör, Theorem 5.6.2] to approximate the family $\xi_{t}^{j}$, uniformly on $f_{0}(K)$, by a family of global holomorphic sections $\tilde{\xi}_{t}^{j}: \widetilde{X} \rightarrow \widetilde{E}$, and we set $\tilde{\xi}_{t}(z)=\sum_{j=1}^{k} g_{j}(h(z)) \tilde{\xi}_{t}^{j}(z)$. The sections

$$
\tilde{f}_{t}(x)=s\left(\tilde{\xi}_{t}\left(f_{0}(x)\right)\right) \in Z \quad\left(x \in X, t \in\left[0, t_{1}\right]\right)
$$

then satisfy theorem 2.1 for $t \in\left[0, t_{1}\right]$.

Using $\tilde{f}_{t_{1}}$ as the new initial global section and repeating the above construction, we obtain a $t_{2}>t_{1}$ and a family of approximating sections $\tilde{f}_{t}: X \rightarrow Z$ for $t \in\left[t_{1}, t_{2}\right]$. We can see as in theorem 4.1 in [FP1] that the proof can be completed in a finite number of steps, their number depending only on the initial family $f_{t}$.

We now state the analogous approximation result for families of sections. If $\left(P, P_{0}\right)$ is a pair of compact Hausdorff spaces as in definition 3 (sect. 1), we set

$$
\widetilde{P}=P \times[0,1], \quad \widetilde{P}_{0}=(P \times\{0\}) \cup\left(P_{0} \times[0,1]\right) .
$$

For each $\widetilde{P}$-section $f: X \times P \times[0,1] \rightarrow Z$ (def. 3) we write $f_{t}=f(\cdot, \cdot, t): X \times P \rightarrow Z$ and $f_{p, t}=f(\cdot, p, t): X \rightarrow Z$.

2.2 Theorem. Let $h: Z \rightarrow X, X_{0}$ and $K$ be as in theorem 2.1, and let $U, V$ be open subsets of $X$ with $K \subset U \subset V \subset \subset X$. Assume that $f: U \times \widetilde{P} \rightarrow Z$ is a holomorphic $\widetilde{P}$-section (def. 3) such that

(i) $f_{p, t}$ extends to a global holomorphic section on $X$ for all $(p, t) \in \widetilde{P}_{0}$ (2.1), and

(ii) $f_{p, t}(x)=f_{p, 0}(x)$ for all $x \in X_{0}$ and $(p, t) \in \widetilde{P}$.

Let $d$ be a metric on $Z$ compatible with the manifold topology. If the submersion $h: Z \rightarrow X$ admits a globally defined spray (def. 2), then for each $\epsilon>0$ there exist a neighborhood $U^{\prime} \supset K$ of $K$ and a holomorphic $\widetilde{P} \times[0,1]=P \times[0,1]^{2}$-section $g: U^{\prime} \times P \times[0,1]^{2} \rightarrow Z$ such that, writing $g^{u}=g(\cdot, \cdot, \cdot, u)$ and $g_{p, t}^{u}=g(\cdot, p, t, u): U^{\prime} \rightarrow Z$, we have

(a) $g^{0}=f$,

(b) $g^{1}$ extends to a holomorphic $\widetilde{P}$-section $\tilde{f}: V \times \widetilde{P} \rightarrow Z$ over $V$,

(c) $g_{p, t}^{u}$ is independent of $u \in[0,1]$ when $(p, t) \in \widetilde{P}_{0}$,

(d) $g_{p, t}^{u}(x)=f_{p, 0}(x)$ for all $x \in X_{0}$ and $(p, t, u) \in P \times[0,1]^{2}$, and 
(e) $d\left(g_{p . t}^{u}(x), f_{p, 0}(x)\right)<\epsilon$ for all $x \in K$ and $(p, t, u) \in P \times[0,1]^{2}$.

Theorem 2.2 is proved by following the proof of theorem 4.2 in [FP1] with the obvious modifications, indicated in the proof of theorem 2.1 above, to insure that we keep everything fixed on the subvariety $X_{0}$. We omit the obvious details.

\section{\&3. Splitting of holomorphic functions on Cartan pairs.}

The main results in this section are lemmas 3.2 and 3.3 which are needed in sect. 4 . We shall use the following notation. $\mathcal{H}(X)$ denotes the Fréchet algebra of holomorphic functions on a complex manifold $X$, equipped with the topology of uniform convergence on compact sets. Any closed complex subvariety $X_{0} \subset X$ carries an induced structure of a reduced complex space, and the space $\mathcal{H}\left(X_{0}\right)$ of all holomorphic functions on $X_{0}$ is also a Fréchet space (see [GuR, p.158, Theorem 5] or [Hör], Corollary 7.2.6). If $Y$ is another complex manifold, $\mathcal{H}(X, Y)$ denotes the space of holomorphic maps $X \rightarrow Y$.

If $D$ is a domain in a complex manifold $X$ and $X_{0}$ is a closed complex subvariety of $X$, we denote by $H_{X_{0}}^{\infty}(D)$ the Banach algebra of bounded holomorphic functions in $D$ which vanish on $X_{0} \cap D$. If $X_{0} \cap D=\emptyset$ we have $H_{X_{0}}^{\infty}(D)=H^{\infty}(D)$. By $H^{\infty}\left(X_{0} \cap D\right)$ we denote the space of bounded holomorphic functions on the subvariety $X_{0} \cap D$.

3.1 Lemma. Let $X$ be a Stein manifold, $X_{0} \subset X$ a closed complex subvariety and $D \subset X$ a pseudoconvex domain in $X$. Then for any relatively compact subdomain $\Omega \subset \subset D$ there exists a bounded linear extension operator $S: H^{\infty}\left(X_{0} \cap D\right) \rightarrow H^{\infty}(\Omega)$ such that $(S f)(x)=x$ for each $f \in H^{\infty}\left(X_{0} \cap D\right)$ and $x \in X_{0} \cap \Omega$.

Remark. If $D \subset \subset X$ is strongly pseudoconvex and if $X_{0}$ has no singularities on $X_{0} \cap b D$ and intersects $b D$ transversely, Henkin ([Hen], [HL1]) constructed a bounded extension operator $S: H^{\infty}\left(X_{0} \cap D\right) \rightarrow H^{\infty}(D)$ (no shrinking of the domain!); for recent related results see $[\mathrm{AAC}]$ and the references therein. No such extension exists in general if $X_{0}$ has singularities along $X_{0} \cap b D$.

Proof. We owe the idea of this proof to Bo Berndtsson (private communication). Since $D$ is pseudoconvex in $X$, the restriction operator $R: \mathcal{H}(D) \rightarrow \mathcal{H}\left(X_{0} \cap D\right)$ is surjective [GuR, p.245, Theorem 18]. Since both spaces are Fréchet (and hence complete), the open mapping theorem applies. Choose a domain $\Omega_{1} \subset X$ such that $\Omega \subset \subset \Omega_{1} \subset \subset D$. By the open mapping theorem the image by $R$ of the set $\left\{f \in \mathcal{H}(D):\|f\|_{L^{\infty}\left(\Omega_{1}\right)}<1\right\}$ contains a neighborhood of the origin in $\mathcal{H}\left(X_{0} \cap D\right)$. This means that there are a relatively compact subset $Y \subset \subset X_{0} \cap D$ and a constant $M<\infty$ such that any $h \in \mathcal{H}\left(X_{0} \cap D\right)$ extends to a function $h^{\prime} \in \mathcal{H}(D)$ satisfying the estimate

$$
\left\|h^{\prime}\right\|_{L^{\infty}\left(\Omega_{1}\right)} \leq M\|h\|_{L^{\infty}(Y)} .
$$

We may assume that $\Omega_{1} \cap X_{0} \subset Y$. The restriction $\left.h^{\prime}\right|_{\Omega_{1}}$ is bounded and hence belongs to the Bergman space $H=L^{2}\left(\Omega_{1}\right) \cap \mathcal{H}\left(\Omega_{1}\right)$, where the $L^{2}$-norm is measured with respect to some smooth hermitian metric on $X$. $H$ is a Hilbert space containing the closed subspace $H_{0}=\left\{f \in H:\left.f\right|_{X_{0}}=0\right\}$. Let $H_{1}$ be the orthogonal complement to $H_{0}$ in $H$. Projecting $h^{\prime}$ 
orthogonally into $H_{1}$ we get a function $\tilde{h} \in H_{1}$ which extends $h$ and which has the minimal $L^{2}\left(\Omega_{1}\right)$ norm among all $L^{2}$-holomorphic extensions of $h$ to $\Omega_{1}$. Clearly such $\tilde{h}$ is unique and $S: h \rightarrow \tilde{h}$ gives a bounded linear operator $S: H^{\infty}\left(X_{0} \cap D\right) \rightarrow L^{2}\left(\Omega_{1}\right)$. Furthermore, restricting $\tilde{h}$ to the subdomain $\Omega \subset \subset \Omega_{1}$ and applying the Cauchy estimates, we get a bounded linear extension operator $S: H^{\infty}\left(X_{0} \cap D\right) \rightarrow H^{\infty}(\Omega)$.

The following lemma is crucial for the results in sections 4 and 5. If $X_{0} \cap \Omega=\emptyset$, lemma 3.2 coincides with lemma 2.4 in [FP1].

3.2 Lemma. Let $X$ be a Stein manifold, $X_{0}$ be a closed complex subvariety of $X$ and $A, B \subset \subset X$ relatively compact domains satisfying the following:

(i) $\Omega=A \cup B$ is a smooth strongly pseudoconvex domain in $X$,

(ii) $\overline{A \backslash B} \cap \overline{B \backslash A}=\emptyset$, and

(iii) $\overline{X_{0} \cap C} \subset \Omega$, where $C=A \cap B$.

Then there exist bounded linear operators $\mathcal{A}: H_{X_{0}}^{\infty}(C) \rightarrow H_{X_{0}}^{\infty}(A), \mathcal{B}: H_{X_{0}}^{\infty}(C) \rightarrow H_{X_{0}}^{\infty}(B)$, such that $c=\mathcal{A}(c)-\mathcal{B}(c)$ on $C$ for each $c \in H_{X_{0}}^{\infty}(C)$.

Proof. Condition (iii) implies that $X_{0} \cap b \Omega \cap \bar{C}=\emptyset$. Hence we can enlarge $\Omega$ slightly around $X_{0} \cap b \Omega$ to get a strongly pseudoconvex domain $\Omega^{\prime} \supset \Omega$ in $X$ satisfying $\Omega^{\prime} \cap b C=\Omega \cap b C$ and $\overline{X_{0} \cap \Omega} \subset X_{0} \cap \Omega^{\prime}$. We can write $\Omega^{\prime}=A^{\prime} \cup B^{\prime}$ where $A^{\prime} \cap B^{\prime}=C, A^{\prime} \supset A, B^{\prime} \supset B$, and the sets $A^{\prime}, B^{\prime}$ also satisfy the separation property (ii). Choose a smooth function $\chi: X \rightarrow[0,1]$ such that $\chi=0$ in an open neighborhood of $\overline{A^{\prime} \backslash B^{\prime}}$ and $\chi=1$ in an open neighborhood of $\overline{B^{\prime} \backslash A^{\prime}}$. For any $c \in H^{\infty}(C)$ the function $\chi c$ extends to a bounded smooth function on $A^{\prime}$ which equals zero outside of $C$, and likewise $(\chi-1) c$ extends to a bounded smooth function on $B^{\prime}$ which equals zero outside of $C$. The difference of these two functions equals $c$ on $C$, but the functions are not holomorphic.

Since $\Omega^{\prime}$ is a relatively compact strongly pseudoconvex domain in a Stein manifold, there exists a linear solution operator $T$ for the $\bar{\partial}$-equation in $\Omega^{\prime}$ which is bounded in the sup-norm, i.e., for any bounded $\bar{\partial}$-closed $(0,1)$-form $g$ on $\Omega^{\prime}$ we have $\bar{\partial}(T g)=g$ and $\|T g\|_{\infty} \leq$ const $\|g\|_{\infty}$ ([HL1], p. 82). Since $\operatorname{supp}(\bar{\partial} \chi) \cap \Omega^{\prime} \subset C$, the bounded $(0,1)$-form $g=\bar{\partial}(\chi c)=\bar{\partial}((\chi-1) c)=c \bar{\partial} \chi$ on $C$ extends to a bounded $(0,1)$-form on $\Omega^{\prime}$ which is zero outside of $C$. Set

$$
a^{\prime}=\left.(\chi c-T(g))\right|_{A^{\prime}}, \quad b^{\prime}=\left.((\chi-1) c-T(g))\right|_{B^{\prime}} .
$$

It is immediate that $a^{\prime} \in H^{\infty}\left(A^{\prime}\right), b^{\prime} \in H^{\infty}\left(B^{\prime}\right)$ and $\left.\left(a^{\prime}-b^{\prime}\right)\right|_{C}=c$. This solves the problem if $X_{0} \cap \Omega=\emptyset$.

Suppose now that $c \in H_{X_{0}}^{\infty}(C)$. The functions $a^{\prime}$ and $b^{\prime}$ need not vanish on $X_{0}$. However, since $\left.\left(a^{\prime}-b^{\prime}\right)\right|_{X_{0} \cap C}=\left.c\right|_{X_{0} \cap C}=0, a^{\prime}$ and $b^{\prime}$ define a function $h \in H^{\infty}\left(X_{0} \cap \Omega^{\prime}\right)$. There exists a pseudoconvex domain $D \subset \subset X$ containing $\Omega^{\prime}$ such that $\bar{\Omega} \subset D$ and $X_{0} \cap D=$ $X_{0} \cap \Omega^{\prime}$. Let $S: H^{\infty}\left(X_{0} \cap D\right) \rightarrow H^{\infty}(\Omega)$ be a bounded linear extension operator provided by lemma 3.1. The pair of functions

$$
a=\left.\left(a^{\prime}-S h\right)\right|_{A} \in H_{X_{0}}^{\infty}(A), \quad b=\left.\left(b^{\prime}-S h\right)\right|_{B} \in H_{X_{0}}^{\infty}(B)
$$


then satisfies lemma 3.2, and every step in the construction was performed by a bounded linear operator between suitable function spaces.

Remark. Lemma 3.2 also holds in spaces of bounded holomorphic functions on $A, B, C$ which vanish to a fixed order $k \in \mathbf{Z}_{+}$along the subvariety $X_{0}$; this could be used to give an alternative proof of the last statement in theorem 1.4 avoiding lemma 3.3. Here is an outline of proof. Let $\mathcal{O}=\mathcal{O}_{X}$ denote the sheaf of germs of holomorphic functions on $X$, and let $\mathcal{J}_{k} \subset \mathcal{O}$ be the sub-sheaf of ideals consisting of germs that vanish to order $k$ along $X_{0}$. We then have a short exact sequence of coherent analytic sheaves on $X$ :

$$
0 \rightarrow \mathcal{J}_{k} \hookrightarrow \mathcal{O} \rightarrow \mathcal{K} \rightarrow 0
$$

where the quotient sheaf $\mathcal{K}=\mathcal{O} / \mathcal{J}_{k}$ is trivial on $X \backslash X_{0}$. Cartan's Theorem B implies that, over any Stein subset $D \subset X$, we can lift any holomorphic section of $\mathcal{K}$ to a holomorphic section of $\mathcal{O}$. If $c \in H^{\infty}(C)$ is a section of $\mathcal{J}_{k}$ over $C$, we get $c=a^{\prime}-b^{\prime}$ with $a^{\prime} \in H^{\infty}\left(A^{\prime}\right)$ and $b^{\prime} \in H^{\infty}\left(B^{\prime}\right)$. The pair $\left(a^{\prime}, b^{\prime}\right)$ defines a holomorphic section $h$ of $\mathcal{K}$ over $\Omega^{\prime}=A^{\prime} \cup B^{\prime}$ which we can lift to a section $\tilde{h}$ of $\mathcal{O}$ over $\Omega^{\prime}$. Then the sections $a=a^{\prime}-\tilde{h}, b=b^{\prime}-\tilde{h}$ solve the problem on $(A, B)$, provided that $h \rightarrow \tilde{h}$ is given by a bounded extension operator as in lemma 3.1. This can be done exactly as before since the space of sections of the sheaf $\mathcal{J}_{k}$ (and hence of $\mathcal{K}$ ) over $\Omega^{\prime}$ is a Fréchet space in the topology of uniform convergence on compact sets ([GuR], Chapter 8).

The next lemma is analogous to lemma 3.2, except that the set $A \subset X$ is not relatively compact. Choose a hermitian metric on $X$ and denote by $d \lambda$ the associated volume element. For any domain $\Omega \subset X$ and plurisubharmonic function $\rho: \Omega \rightarrow \mathbf{R}$ let

$$
H_{\rho}^{2}(\Omega)=\left\{f \in \mathcal{H}(\Omega):\|f\|_{L_{\rho}^{2}(\Omega)}^{2}=\int_{\Omega}|f|^{2} e^{-\rho} d \lambda<\infty\right\}
$$

denote the Bergman space on $\Omega$ with weight $e^{-\rho}$. For any subdomain $D \subset \Omega$ we define

$$
H_{\rho}^{2, \infty}(\Omega, D)=\left\{f \in \mathcal{H}(\Omega):\|f\|_{\rho, \infty}=\|f\|_{L_{\rho}^{2}(\Omega)}+\|f\|_{L^{\infty}(D)}<+\infty\right\}
$$

Clearly this is a Banach space with the norm $\|\cdot\|_{\rho, \infty}$.

3.3 Lemma: Let $X$ be a Stein manifold and $A, B \subset X$ open sets such that $\bar{B}$ is compact. Let $C=A \cap B$. Assume that $\Omega=A \cup B$ is a smooth strongly pseudoconvex domain in $X$ and $\overline{A \backslash B} \cap \overline{B \backslash A}=\emptyset$. For any open subset $D \subset \subset \Omega$ there are a smooth plurisubharmonic function $\rho: \Omega \rightarrow \mathbf{R}$ and bounded linear operators

$$
\mathcal{A}: H^{\infty}(C) \rightarrow H_{\rho}^{2, \infty}(A, A \cap D), \quad \mathcal{B}: H^{\infty}(C) \rightarrow H^{\infty}(B)
$$

satisfying $c=\mathcal{A}(c)-\mathcal{B}(c)$ on $C$ for each $c \in H^{\infty}(C)$.

Proof. Since we may enlarge $D \subset \Omega$ without affecting the statement of lemma 3.3, we may assume that $D=D^{\prime} \cap \Omega$ for some domain $D^{\prime} \subset \subset X$ satisfying $\bar{B} \subset D^{\prime}$. Choose domains 
$D_{0}^{\prime}, D_{1}^{\prime} \subset \subset X$ such that $D^{\prime} \subset \subset D_{0}^{\prime} \subset \subset D_{1}^{\prime}$ and set $D_{j}=\Omega \cap D_{j}^{\prime} \subset \Omega$ for $j=0,1$. We may assume that $D_{1}$ is strongly pseudoconvex. Let $T$ be a linear, sup-norm bounded solution operator for the $\bar{\partial}$-equation in $D_{1}$ ([HL1], p. 82). Choose a cut-off function $\chi: X \rightarrow[0,1]$ as in the proof of lemma 3.2 and take

$$
\begin{aligned}
a^{\prime} & =\left.(\chi c-T(c \bar{\partial} \chi))\right|_{A \cap D_{1}} \in H^{\infty}\left(A \cap D_{1}\right), \\
b^{\prime} & =\left.((\chi-1) c-T(\bar{\partial} \chi))\right|_{B} \in H^{\infty}(B) .
\end{aligned}
$$

We have $\left.\left(a^{\prime}-b^{\prime}\right)\right|_{C}=c$, but $a^{\prime}$ is only defined on $A \cap D_{1}$. To correct this we shall solve another $\bar{\partial}$-equation as follows. Since $\Omega$ is smooth strongly pseudoconvex, we can enlarge it slightly within $D_{0}^{\prime}$ to obtain a smooth strongly pseudoconvex domain $\Omega^{\prime} \subset X$ satisfying $\Omega \cup \bar{D} \subset \Omega^{\prime}$ and $\Omega \backslash D_{0}^{\prime}=\Omega^{\prime} \backslash D_{0}^{\prime}$. Choose a smooth cut-off function $\tau: X \rightarrow[0,1]$ such that $\tau=1$ in a neighborhood of $\bar{D}_{0}^{\prime}$ and $\operatorname{supp} \tau \subset \subset D_{1}^{\prime}$. Hence $\operatorname{supp}(\bar{\partial} \chi) \cap \Omega^{\prime} \subset D_{1} \backslash \bar{D}_{0}$. The $\bar{\partial}$-closed $(0,1)$-form $g=\bar{\partial}\left(\tau a^{\prime}\right)=a^{\prime} \bar{\partial} \tau$, defined initially on $D_{1} \backslash \bar{D}_{0}$, extends to a bounded form on $\Omega^{\prime}$ which is zero outside $D_{1} \backslash \bar{D}_{0}$. By Hörmander [Hör] there is a smooth plurisubharmonic function $\rho: \Omega^{\prime} \rightarrow[0,+\infty)$ such that the equation $\bar{\partial} u=g$ has a smooth solution $u$ in $\Omega^{\prime}$ satisfying $\|u\|_{L_{\rho}^{2}\left(\Omega^{\prime}\right)} \leq$ const $\|g\|_{\infty} \leq$ const $\|c\|_{L^{\infty}(C)}$. Moreover, if we take $u$ to be the (unique) solution with minimal $L_{\rho}^{2}\left(\Omega^{\prime}\right)$ norm, the composition $c \rightarrow g \rightarrow u$ defines a bounded linear operator $H^{\infty}(C) \rightarrow L_{\rho}^{2}\left(\Omega^{\prime}\right)$. By a well known estimate on the interior regularity of the $\bar{\partial}$-operator we have for any compact subset $K \subset \Omega^{\prime}$

$$
\|u\|_{L^{\infty}(K)} \leq \operatorname{const}\left(\|u\|_{L_{\rho}^{2}\left(\Omega^{\prime}\right)}+\|\bar{\partial} u\|_{L^{\infty}\left(\Omega^{\prime}\right)}\right) .
$$

Applying this estimate on the compact subsets $\bar{B}, \bar{D} \subset \Omega^{\prime}$ we see that

$$
a=\left.\left(\tau a^{\prime}-u\right)\right|_{A} \in H_{\rho}^{2, \infty}(A, A \cap D), \quad b=\left.\left(b^{\prime}-u\right)\right|_{B} \in H^{\infty}(B) .
$$

Clearly $\left.(a-b)\right|_{C}=c$, and the maps $c \rightarrow a, c \rightarrow b$ for $c \in H^{\infty}(C)$ are bounded linear operators into the respective Banach spaces.

\section{\&4. Attaching lemma: the model case.}

In this section we apply lemmas 3.2 and 3.3 to construct holomorphic sections of certain model fibrations. An iteration scheme to solve this problem was proposed in [Gro]; we shall apply the implicit function theorem in suitable function spaces.

Recall that a pair of open subset $U \subset V$ in a complex manifold $X$ is a Runge pair, or $U$ is Runge in $V$, if every holomorphic function in $U$ can be approximated, uniformly on compacts in $U$, by functions holomorphic in $V$. We use the notation introduced in sect. 3 above, and we identify the subvariety $X_{0} \subset X$ with $X_{0} \times\{0\} \subset X \times \mathbf{C}^{n}$.

4.1 Proposition. Let $X$ be a Stein manifold, $X_{0}$ a closed complex subvariety of $X$ and $A, B \subset \subset X$ relatively compact open subsets as in lemma 3.2. Let $\widetilde{B} \supset \bar{B}$ and $\widetilde{C} \supset \bar{C}$ be open sets such that $\widetilde{C} \subset \widetilde{B}$ and $\widetilde{C}$ is Runge in $\widetilde{B}$. Let $U \subset \mathbf{C}^{n}$ be an open neighborhood of the origin and $\psi_{0}: \widetilde{C} \times U \rightarrow \mathbf{C}^{n}$ a bounded holomorphic map such that for each $x \in$ 
$\widetilde{C}$ we have $\psi_{0}(x, 0)=0$ and $\psi_{0}(x, \cdot): U \rightarrow \mathbf{C}^{n}$ is injective. Then there exist an open neighborhood $\mathcal{W}$ of $\psi_{0}$ in the Banach space $H_{X_{0}}^{\infty}(\widetilde{C} \times U)^{n}$ and smooth Banach space operators $\mathcal{A}^{\prime}: \mathcal{W} \rightarrow H_{X_{0}}^{\infty}(A)^{n}, \mathcal{B}^{\prime}: \mathcal{W} \rightarrow H_{X_{0}}^{\infty}(B)^{n}$, with $\mathcal{A}^{\prime}\left(\psi_{0}\right)=0$ and $\mathcal{B}^{\prime}\left(\psi_{0}\right)=0$, such that for each $\psi \in \mathcal{W}$ the bounded holomorphic maps $\alpha=\mathcal{A}^{\prime}(\psi): A \rightarrow \mathbf{C}^{n}$ and $\beta=\mathcal{B}^{\prime}(\psi): B \rightarrow \mathbf{C}^{n}$ satisfy $\left.\alpha\right|_{A \cap X_{0}}=0,\left.\beta\right|_{B \cap X_{0}}=0$ and

$$
\psi(x, \alpha(x))=\beta(x) \quad(x \in C=A \cap B) .
$$

Moreover, if $\psi \in \mathcal{W}$ satisfies $\psi(x, 0)=0$ for $x \in \widetilde{C}$, then $\mathcal{A}^{\prime}(\psi)=0$ and $\mathcal{B}^{\prime}(\psi)=0$.

Remark. We can view a pair of maps satisfying (4.1) as a section of the fibration over $A \cup B \subset X$ obtained by identifying the point $(x, z) \in C \times U \subset A \times \mathbf{C}^{n}$ with the point $(x, \psi(x, z)) \in C \times \mathbf{C}^{n}$. For applications to parametrized families it is convenient to have solutions given by operators, although this could be avoided by a suitable analogue of Satz 8 in [Gr1].

Proof. We apply the proof of proposition 5.2 in [FP1], replacing lemma 2.4 in [FP1] by lemma 3.2 above. We recall the main idea and refer to [FP1] for the details. Assume first $\psi_{0}(x, z)=z(x \in \widetilde{C}, z \in U)$. Let $\mathcal{A}: H_{X_{0}}^{\infty}(C)^{n} \rightarrow H_{X_{0}}^{\infty}(A)^{n}$ and $\mathcal{B}: H_{X_{0}}^{\infty}(C)^{n} \rightarrow H_{X_{0}}^{\infty}(B)^{n}$ be the linear operators obtained by applying lemma 3.2 componentwise; hence $c=\mathcal{A} c-\mathcal{B} c$ for all $c \in H_{X_{0}}^{\infty}(C)^{n}$. Consider the operator

$$
\begin{aligned}
& \Phi: H_{X_{0}}^{\infty}(\widetilde{C} \times U)^{n} \times H_{X_{0}}^{\infty}(C)^{n} \rightarrow H_{X_{0}}^{\infty}(C)^{n}, \\
& \Phi(\psi, c)(x)=\psi(x, \mathcal{A} c(x))-\mathcal{B} c(x) \quad(x \in C) .
\end{aligned}
$$

This is a smooth Banach space operator in a neighborhood of the point $\left(\psi_{0}, 0\right)$, satisfying $\Phi\left(\psi_{0}, c\right)=\mathcal{A} c-\mathcal{B} c=c$. By the implicit function theorem the equation $\Phi(\psi, c)=0$ has locally near $\left(\psi_{0}, 0\right)$ a unique solution $c=\mathcal{C}(\psi)$ given by a smooth operator $\mathcal{C}$. The operators $\mathcal{A}^{\prime}=\mathcal{A} \circ \mathcal{C}, \mathcal{B}^{\prime}=\mathcal{B} \circ \mathcal{C}$ then satisfy proposition 4.1. The general case is reduced to this one by approximating $\psi_{0}$ by a holomorphic map on $\widetilde{B} \times U$ (see [FP1]).

Applying lemma 3.3 instead of lemma 3.2 in the proof of proposition 4.1 we get the following result.

4.2 Proposition. Let $X$ be a Stein manifold and let $A, B \subset X$ and $D \subset \Omega=A \cup B$ be open subsets as in lemma 3.3. Let $\widetilde{B} \supset \bar{B}$ and $\widetilde{C} \supset \bar{C}$ be open sets such that $\widetilde{C} \subset \widetilde{B}$ and $\widetilde{C}$ is Runge in $\widetilde{B}$. Let $U \subset \mathbf{C}^{n}$ be an open neighborhood of the origin and $\psi_{0}: \widetilde{C} \times U \rightarrow \mathbf{C}^{n}$ a bounded holomorphic map such that for each $x \in \widetilde{C}, \psi_{0}(x, 0)=0$ and $\psi_{0}(x, \cdot): U \rightarrow \mathbf{C}^{n}$ is injective. Then there exist an open neighborhood $\mathcal{W} \subset H^{\infty}(\widetilde{C} \times U)^{n}$ of $\psi_{0}$ and smooth Banach space operators $\mathcal{A}^{\prime}: \mathcal{W} \rightarrow H_{\rho}^{2, \infty}(A, A \cap D)^{n}, \mathcal{B}^{\prime}: \mathcal{W} \rightarrow H^{\infty}(B)^{n}$, with $\mathcal{A}^{\prime}\left(\psi_{0}\right)=0$ and $\mathcal{B}^{\prime}\left(\psi_{0}\right)=0$, such that for each $\psi \in \mathcal{W}$ the holomorphic maps $\alpha=\mathcal{A}^{\prime}(\psi): A \rightarrow \mathbf{C}^{n}$ and $\beta=\mathcal{B}^{\prime}(\psi): B \rightarrow \mathbf{C}^{n}$ satisfy (4.1). Moreover, if $\psi \in \mathcal{W}$ satisfies $\psi(x, 0)=0$ for all $x \in \widetilde{C}$, then $\mathcal{A}^{\prime}(\psi)=0$ and $\mathcal{B}^{\prime}(\psi)=0$. 


\section{\&5. Attaching lemma for holomorphic sections of submersions.}

In this sections we use the results of sect. 4 in order patch together holomorphic sections of a submersion $Z \rightarrow X$ over a Cartan pair $(A, B)$ in $X$, provided that the two sections are sufficiently close on the intersection $A \cap B$. Theorem 5.1 below is similar to results in sect. 1.6 of [Gro] and to theorems 5.1 and 5.5 in [FP1]; the additional point here is the interpolation on a subvariety $X_{0} \subset X$. Theorem 5.2 is new and depends on proposition 4.2 .

We recall from [FP2] the definition of a Cartan pair. (Note that our earlier definition in [FP1] did not include the Runge property (iii).)

Definition 4. An ordered pair of compact sets $(A, B)$ in a complex manifold $X$ is said to be a Cartan pair if

(i) each of the set $A, B$, and $A \cup B$ has a basis of Stein neighborhoods,

(ii) $\overline{A \backslash B} \cap \overline{B \backslash A}=\emptyset \quad$ (separation condition), and

(iii) the set $C=A \cap B$ is Runge in $B$. ( $C$ may be empty.)

If in addition $X_{0}$ is a closed complex subvariety of $X$ such that $X_{0} \cap C \subset \operatorname{Int}(A \cup B)$, we shall say that the Cartan pair $(A, B)$ is $X_{0}$-regular.

We proved in [FP1] that for each Cartan pair $(A, B)$ in $X$ there exist bases of decreasing open neighborhoods $A_{j} \supset A, B_{j} \supset B\left(j \in \mathbf{Z}_{+}\right)$such that each pair $\left(A_{j}, B_{j}\right)$ satisfies the hypothesis (i) and (ii) of lemma 3.2 and $C_{j}$ is Runge in $B_{j}$. The same proof shows that, if $(A, B)$ is $X_{0}$-regular, we also get property (iii) in lemma 3.2 for each $\left(A_{j}, B_{j}\right)$. Hence proposition 4.1 can be applied on a suitable bases of neighborhoods of any $X_{0}$-regular Cartan pair $(A, B)$ in $X$. This allows us to glue sections of submersions $h: Z \rightarrow X$ over $(A, B)$ when $Z$ admits a spray over a neighborhood of $B$.

The presence of parameters and the need to do everything by homotopies complicates the statement, so let us first explain the result in the basic case. Let $(A, B)$ be an $X_{0^{-}}$ regular Cartan pair in $X$. We are given holomorphic sections $a: \widetilde{A} \rightarrow Z$ resp. $b: \widetilde{B} \rightarrow Z$ over open sets $\widetilde{A} \supset A$ resp. $\widetilde{B} \supset B$ such that $a$ and $b$ agree on $X_{0} \cap \widetilde{C}$, where $\widetilde{C}=\widetilde{A} \cap \widetilde{B}$. If $Z$ admits a spray over $\widetilde{B}$ and if $\tilde{b}$ is sufficiently close to $\tilde{a}$ on $\widetilde{C}$, we can move each of the two sections a little (by holomorphic homotopies of sections on $\widetilde{A}$ resp. $\widetilde{B}$ ) such that the final pair of sections coincides on $\widetilde{A} \cap \widetilde{B}$ and hence gives a holomorphic section over $\widetilde{A} \cup \widetilde{B}$. (We must shrink the neighborhoods of $A$ and $B$ in the process.) Moreover, we can perform the procedure so that the homotopy is fixed on $X_{0}$ where the initial sections already agree. The same can be done for $P$-sections so that the homotopies are fixed for those values of the parameter for which the two initial sections already coincide over $\widetilde{C}$.

Having said this, we state the result in precise terms.

5.1 Theorem. Let $h: Z \rightarrow X$ be a holomorphic submersion onto a Stein manifold $X$, let $X_{0}$ be a closed complex subvariety of $X$, and let $(A, B)$ be a $X_{0}$-regular Cartan pair in $X$ (def. 4). Suppose that $\widetilde{B}$ is an open neighborhood of $B$ in $X$ such that the restriction $\left.Z\right|_{\widetilde{B}}=h^{-1}(\widetilde{B})$ admits a spray over $\widetilde{B}$ (def. 2). Let $\left(P, P_{0}\right)$ be as in def. 3. Let $\widetilde{A} \supset A$ be an open neighborhood of $A$ in $X$ and $a: \widetilde{A} \times P \rightarrow Z$ a holomorphic P-section over $\widetilde{A}$ (def. 
3). Fix a metric $d$ on $Z$ compatible with the manifold topology. Then for each $\epsilon>0$ there is a $\delta>0$ satisfying the following property. If $b: \widetilde{B} \times P \rightarrow Z$ is a holomorphic P-section over $\widetilde{B}$ satisfying

$$
\begin{array}{ll}
d\left(a_{p}(x), b_{p}(x)\right)<\delta & (x \in \widetilde{C}=\widetilde{A} \cap \widetilde{B}, p \in P), \\
a_{p}(x)=b_{p}(x) & \left(x \in \widetilde{C}, p \in P_{0}\right) \text { or }\left(x \in X_{0} \cap \widetilde{C}, p \in P\right),
\end{array}
$$

then there exist smaller neighborhoods $A^{\prime} \supset A, B^{\prime} \supset B$ and homotopies of holomorphic $P$-sections $a^{t}: A^{\prime} \times P \rightarrow Z, b^{t}: B^{\prime} \times P \rightarrow Z(t \in[0,1])$ such that

(a) $a_{p}^{t}=a_{p}$ and $b_{p}^{t}=b_{p}$ for $(p, t) \in \widetilde{P}_{0}=(P \times\{0\}) \cup\left(P_{0} \times[0,1]\right)$,

(b) $a_{p}^{t}(x)=a_{p}(x)$ and $b_{p}^{t}(x)=b_{p}(x)$ for $x \in X_{0}$ and $(p, t) \in P \times[0,1]$,

(c) $a_{p}^{1}(x)=b_{p}^{1}(x)$ for $x \in C^{\prime}=A^{\prime} \cap B^{\prime}$ and $p \in P$, and

(d) for each $(p, t) \in P \times[0,1]$ we have

$$
d\left(a_{p}^{t}(x), a_{p}(x)\right)<\epsilon \quad\left(x \in A^{\prime}\right), \quad d\left(b_{p}^{t}(x), b_{p}(x)\right)<\epsilon \quad\left(x \in C^{\prime}\right) .
$$

Remark. Property (c) implies that $a^{1}$ and $b^{1}$ together define a holomorphic $P$-section $\tilde{a}:\left(A^{\prime} \cup B^{\prime}\right) \times P \rightarrow Z$ over $A^{\prime} \cup B^{\prime}$. For $p \in P_{0}$ the section $\tilde{a}_{p}$ agrees with $a_{p}$ and $b_{p}$ according to (a), and the homotopy is fixed on $X_{0}$ according to (b).

Proof. It suffices to apply the proof of theorem 5.5 in [FP1], but replacing proposition 5.2 in [FP1] by proposition 3.2 above. We recall the main idea since we shall need this in the next theorem. Suppose for simplicity that $P$ is a singleton and $P_{0}=\emptyset$. We linearize the problem by first constructing for some large $n \in \mathbf{Z}_{+}$a pair of holomorphic maps

$$
s_{1}: V \subset \widetilde{A} \times \mathbf{C}^{n} \rightarrow Z, \quad s_{2}: \widetilde{B} \times \mathbf{C}^{n} \rightarrow Z,
$$

where $V$ is an open set containing $\widetilde{A} \times\{0\}$, such that $s_{1}$ and $s_{2}$ are fiber preserving (i.e., the fiber over $x \in \widetilde{A}$ resp. $x \in \widetilde{B}$ is mapped into $Z_{x}=h^{-1}(x)$ ), and they are submersions of open neighborhoods of the zero sections in $\widetilde{A} \times \mathbf{C}^{n}$ resp. $\widetilde{B} \times \mathbf{C}^{n}$ onto open neighborhoods of the graphs $a(\widetilde{A}) \subset Z$ resp. $b(\widetilde{B}) \subset Z$. Moreover we have $s_{1}(x, 0)=a(x)$ and $s_{2}(x, 0)=b(x)$.

The submersion $s_{1}$ is only locally defined and can be obtained from local flows of vertical holomorphic vector fields on $Z$ (tangent to fibers of $h$ ) near $a(A)$. The second map $s_{2}$ is globally defined and is obtained by restricting the spray map $s:\left.E \rightarrow Z\right|_{\widetilde{B}}$ to the vector bundle $\left.E\right|_{b(\widetilde{B})}$ over the section $b(\widetilde{B}) \subset Z$. The total space $\left.E\right|_{b(\widetilde{B})}$ is not necessarily trivial; however, we may choose $\widetilde{B}$ to be Stein, and hence there is a holomorphic vector bundle epimorphism $\theta: \widetilde{B} \times\left.\mathbf{C}^{n} \rightarrow E\right|_{b(\widetilde{B})}$ for any sufficiently large $n$. The composition $s_{2}=s \circ \theta$ satisfies our requirements.

When $a$ and $b$ are sufficiently close to each other over $\widetilde{C}$, we can construct a holomorphic transition map $\psi: \widetilde{C} \times U \rightarrow \mathbf{C}^{n}$ as in proposition 4.1 such that

$$
s_{1}(x, z)=s_{2}(x, \psi(x, z)) \quad\left(x \in \widetilde{C}, z \in U \subset \mathbf{C}^{n}\right) .
$$


The closeness of $a$ and $b$ over $\widetilde{C}$ implies that $\psi$ is close to a map $\psi_{0}$ which preserves zero section: $\psi_{0}(x, 0)=0$ for $x \in \widetilde{C}$. If $\alpha: A^{\prime} \rightarrow U \subset \mathbf{C}^{n}$ and $\beta: B^{\prime} \rightarrow \mathbf{C}^{n}$ are holomorphic maps on open sets $A^{\prime} \subset \widetilde{A}$ resp. $B^{\prime} \subset \widetilde{B}$ as in proposition 4.1 , satisfying $\psi(x, \alpha(x))=\beta(x)$ for $x \in C^{\prime}=A^{\prime} \cap B^{\prime}$, we set for each $t \in[0,1]$

$$
a^{t}(x)=s_{1}(x, t \alpha(x)) \quad\left(x \in A^{\prime}\right), \quad b^{t}(x)=s_{2}(x, t \beta(x)) \quad\left(x \in B^{\prime}\right) .
$$

Then $a^{0}=a, b^{0}=b$, and $a^{1}(x)=b^{1}(x)$ for $x \in C^{\prime}$; hence $a^{1}$ and $b^{1}$ together define a holomorphic section $\tilde{a}: A^{\prime} \cup B^{\prime} \rightarrow Z$. The homotopies $a^{t}$ and $b^{t}$ are fixed on $X_{0}$. The details can be found in [FP1].

We need a similar result obtained from proposition 4.2. For simplicity we state the result without parameters (when $P$ is a singleton), even though the result holds in the same generality as theorem 5.1 above.

Theorem 5.2. Let $h: Z \rightarrow X$ be a holomorphic submersion onto a Stein manifold $X$ and let $X_{0}$ be a closed complex subvariety of $X$. Furthermore let $A \subset X, B \subset X$ be closed subsets such that $B$ is compact while $A$ contains a neighborhood of $X_{0}$. Assume that

(i) $A \cup B$ is (the closure of) a strongly pseudoconvex domain in $X$,

(ii) $\overline{A \backslash B} \cap \overline{B \backslash A}=\emptyset$, and

(iii) the set $C=A \cap B$ is Runge in $B$ and $C \cap X_{0}=\emptyset$.

Assume that $Z$ admits a spray over an open set $\widetilde{B} \supset B$. Let $\widetilde{A} \supset A$ be an open set and $a: \widetilde{A} \rightarrow Z$ a holomorphic section. Fix a metric $d$ on $Z$ compatible with the manifold topology. Let $D \subset \subset X$ be an arbitrary relatively compact subset containing $\bar{B}$. Choose an integer $k \in \mathbf{Z}_{+}$. Then for each $\epsilon>0$ there is a $\delta>0$ satisfying the following property. If $b: \widetilde{B} \rightarrow Z$ is a holomorphic section satisfying $d(a(x), b(x))<\delta$ for $x \in \widetilde{C}=\widetilde{A} \cap \widetilde{B}$, there exist open sets $A^{\prime}, B^{\prime} \subset X$ satisfying $X_{0} \cup(A \cap D) \subset A^{\prime} \subset \widetilde{A}, B \subset B^{\prime} \subset \widetilde{B}$, and homotopies of holomorphic sections $a^{t}: A^{\prime} \rightarrow Z, b^{t}: B^{\prime} \rightarrow Z(t \in[0,1])$ such that

(a) $a^{0}=a$ and $b^{0}=b$,

(b) $a^{1}(x)=b^{1}(x)$ for $x \in C^{\prime}=A^{\prime} \cap B^{\prime}$,

(c) for each $t \in[0,1], a^{t}$ agrees with $a=a^{0}$ to order $k$ along $X_{0}$ and satisfies

$$
d\left(a^{t}(x), a(x)\right)<\epsilon \quad\left(x \in A^{\prime} \cap D\right), \quad d\left(b^{t}(x), b(x)\right)<\epsilon \quad\left(x \in C^{\prime}\right) .
$$

Proof. The proof is essentially the same as in theorem 5.1 except for the construction of the map $s_{1}$ (5.1). As in theorem 5.1 we construct a preliminary fiber-preserving holomorphic submersion $\tilde{s}_{1}: V \rightarrow Z$ from an open set $V \subset \widetilde{A} \times \mathbf{C}^{n}$, with $\widetilde{A} \times\{0\} \subset V$, onto a neighborhood of $a(\widetilde{A})$ in $Z$. By the Oka-Cartan theory there exist finitely many holomorphic functions $h_{j}: X \rightarrow \mathbf{C}(1 \leq j \leq m)$ such that $X_{0}=\left\{x \in X: h_{j}(x)=0,1 \leq\right.$ $j \leq m\}$ and each $h_{j}$ vanishes to order $k$ on $X_{0}$. Let $g: \widetilde{A} \times \mathbf{C}^{n m} \rightarrow \mathbf{C}^{n}$ be the map $g\left(x, v_{1}, \ldots, v_{m}\right)=\sum_{j=1}^{m} h_{j}(x) v_{j}$, where $x \in \widetilde{A}$ and $v_{j} \in \mathbf{C}^{n}$ for each $j$. Clearly $g(x, \cdot)$ is 
a linear epimorphism for each $x$ outside $X_{0} \cap \widetilde{A}$ and is degenerate for $x \in X_{0} \cap \widetilde{A}$. Set $s_{1}=\tilde{s}_{1} \circ(I d, g): \widetilde{A} \times \mathbf{C}^{N} \rightarrow Z$, where $I d$ indicates the identity on $\widetilde{A}$ and $N=n m$. Then $s_{1}$ is a submersion on a neighborhood of $\left(\widetilde{A} \backslash X_{0}\right) \times\{0\}$ in $\widetilde{A} \times \mathbf{C}^{N}$ (and is degenerate over $\left.X_{0} \cap \widetilde{A}\right)$. We construct a map $s_{2}: \widetilde{B} \times \mathbf{C}^{N} \rightarrow Z(5.1)$ as before.

Since $C=A \cap B$ does not intersect $X_{0}$, we may assume that the closure of $\widetilde{C}=\widetilde{A} \cap \widetilde{B}$ does not intersect $X_{0}$ either. Hence $s_{1}$ is a submersion over $\widetilde{C}$ which allows us to construct the transition map $\psi(5.2)$ as before. For any solution $(\alpha, \beta)$ of the equation (4.1) we get the corresponding pair of sections

$$
a^{1}(x)=s_{1}(x, \alpha(x))=\tilde{s}_{1}(x, g(x, \alpha(x))), \quad b^{1}(x)=s_{2}(x)
$$

as in (5.3) which agree on a neighborhood of $C$. The section $b^{1}$ is defined on a neighborhood $B^{\prime} \supset B$ as before. On the other hand, the domain of $a^{1}(x)$ may shrink because we cannot control the sup-norm of $\alpha$ on all of $A$ (since $A$ is unbounded), and hence the section $\tilde{\alpha}(x)=(x, g(x, \alpha(x))) \in \widetilde{A} \times \mathbf{C}^{n}$ may escape from the domain of $\tilde{s}_{1}$. However, things are not too bad. We can control the sup-norm of $\alpha$, and hence of $\tilde{\alpha}$, on $A \cap D$ for any $D \subset \subset X$. By construction the map $x \rightarrow g(x, \alpha(x))=\sum_{j} h_{j}(x) \alpha_{j}(x)$ vanishes to order $k$ on $X_{0}$; hence $X_{0}$ is in the domain of $a^{1}$ as well. It follows that $a^{1}$ is holomorphic on a set $A^{\prime} \supset X_{0} \cup(A \cap D)$ and it agrees with $a=a^{0}$ to order $k$ along $X_{0}$. The same applies to each section in the homotopy $a^{t}$ from $a=a^{0}$ to $a^{1}$, and this insures the validity of property (c). All the rest is the same as in theorem 5.1; in particular we get uniform approximation in (d) from the uniform estimates on $A \cap D$ in proposition 4.2.

\section{\&6. Proof of propositions 1.2, 1.3 and theorem 1.4.}

Proof of proposition 1.2. We embed $Y$ as a closed complex submanifold of some Euclidean space $\mathbf{C}^{N}$. By Docquier and Grauert ([GuR], p.257, Theorem 8) there is a holomorphic retraction $\pi: U \rightarrow Y$ of an open neighborhood $U \subset \mathbf{C}^{N}$ onto $Y$. We identify the holomorphic tangent bundle $X=T Y$ with a subbundle of $\left.T \mathbf{C}^{N}\right|_{Y}$ and we identify $Y$ with the zero section of $T Y$. Denote the points in $T Y$ by $(y, \xi)$ and let $0_{y}=(y, 0)$. There is an open neighborhood $V \subset T Y$ of the zero section on which the map $s_{0}(y, \xi)=\pi(x+\xi) \in Y$ is defined and holomorphic. By modifying $s_{0}$ outside a neighborhood of the zero section we can extend it to a smooth map $s_{0}: T Y \rightarrow Y$. Clearly the derivative $d s_{0}: T_{0_{y}}(T Y) \rightarrow T_{y} Y$ restricts to the identity map on $T_{y} Y \subset T_{0_{y}}(T Y)$ for each $y \in Y$. Hence $s_{0}$ satisfies the requirements for a spray, except that it is not globally holomorphic on $T Y$. Observe that $X=T Y$ is a Stein manifold and $X_{0}=Y$ (the zero section) is a closed complex submanifold of $X$. If $s: T Y \rightarrow Y$ is a holomorphic map which matches $s_{0}$ to the second order along the zero section (such $s$ exists by the hypothesis in proposition 1.2), then $s$ is a spray on $Y$.

Proof of proposition 1.3. The image $Z_{0}=f_{0}\left(X_{0}\right)$ is a closed Stein subspace of $Z$ and hence it has an open Stein neighborhood in $Z$ according to [Siu]. The same applies to $X_{0}$ in $X$; hence we may assume that both $X$ and $Z$ are Stein. For each $g \in \mathcal{O}(Z)$ we denote by $d^{\prime} g$ the restriction of the differential $d g$ to the vertical bundle $V T(Z)$. By the 
Oka-Cartan's theory there are functions $g_{1}, \ldots, g_{d} \in \mathcal{O}(Z)$ such that $g_{j}=0$ on $Z_{0}$ for each $j$ and $\left\{d^{\prime} g_{j}: 1 \leq j \leq d\right\}$ span the vertical contangent space $V T_{z}^{*}(Z)$ at each point $z \in Z_{0}$. Hence the map

$$
G: Z \rightarrow X \times \mathbf{C}^{d}, \quad G(z)=\left(h(z), g_{1}(z), \ldots, g_{d}(z)\right)
$$

embeds a neighborhood of $Z_{0}$ as a closed complex submanifold $W$ in an open set $V \subset$ $X \times \mathbf{C}^{d}$, with $G\left(Z_{0}\right)=X_{0} \times\{0\}^{d}$. Denoting by $p: X \times \mathbf{C}^{d} \rightarrow X$ the projection onto $X$, we have $p \circ G=h$.

We claim that, after shrinking $V$ around $W$, we can find a holomorphic retraction $\pi: V \rightarrow W$ satisfying $p \circ \pi=p$. Such a retraction is constructed as in the DocquierGrauert theorem ([GuR], p.257, Theorem 8); here is a brief outline. Let $V T(W) \rightarrow W$ be the vertical tangent bundle to $W$ (with respect to $p: W \rightarrow X$ ); observe that $V T(W)$ is a subbundle of the trivial bundle $W \times \mathbf{C}^{d}$. Choose a complementary holomorphic vector bundle $H \rightarrow W$ such that $H \oplus V T(W)=W \times \mathbf{C}^{d}$. Denote the points of $H$ by $(x, w, \xi)$, with $(x, w) \in W$ and $\xi \in H_{(x, w)} \subset \mathbf{C}^{d}$. Consider the map $H \rightarrow X \times \mathbf{C}^{d}$, $(x, w, \xi) \rightarrow(x, w+\xi)$ (the addition takes place in the fiber $\left.\{x\} \times \mathbf{C}^{d}\right)$. As in the proof of the Docquier-Grauert theorem we see that this map takes a neighborhood of the zero section in $H$ biholomorphically onto a neighborhood of $W$ in $X \times \mathbf{C}^{d}$; hence it conjugates the base projection $H \rightarrow W$ to a desired retraction $V \rightarrow W$.

To extend the initial holomorphic section we take $f(x)=G^{-1}(\pi(x, 0))$, where 0 denotes the origin in $\mathbf{C}^{d}$. When $x$ is sufficiently near $X_{0}$, the point $(x, 0) \in X \times \mathbf{C}^{d}$ belongs to $V$ (the domain of $\pi$ ), and hence $\pi(x, 0) \in W$ belongs to the range of $G$. Thus $f$ is well-defined and holomorphic in an open set $U \supset X_{0}$ in $X$, and we have $f(x) \in Z_{x}$ for $x \in U$. When $x \in X_{0}$, we have $(x, 0) \in W$, hence $\pi(x, 0)=(x, 0)=G\left(f_{0}(x)\right)$ and therefore $f(x)=f_{0}(x)$.

If $f_{0}$ extends continuously to a section $X \rightarrow Z$, we can patch the section $f$ obtained above with $f_{0}$ in a small neighborhood of $X_{0}$ as follows. As above we have an embedding of a neighborhood of $Z_{0}$ in $Z$ as a submanifold $W \subset X \times \mathbf{C}^{d}$; we patch the two sections in the ambient space $X \times \mathbf{C}^{d}$ (where we have linear fibers) by a cut-off function, and finally we project the result back to $W$ by the holomorphic retraction. The new section $f_{1}: X \rightarrow Z$ equals $f$ in a neighborhood of $X_{0}$ (so it is holomorphic there), and it equals $f_{0}$ outside a larger neighborhood of $X_{0}$. The construction also gives a homotopy of $f_{0}$ to $f_{1}$ which is fixed on $X_{0}$. This proves proposition 1.3. The proof carries over verbatim to the case when $X$ is a complex space.

Proof of theorem 1.4. We consider two cases: in the first case the initial continuous section $f_{0}: X \rightarrow Z$ is holomorphic on the subvariety $X_{0}$ and in a neighborhood of a $\mathcal{H}(X)$-convex set $K \subset X$; in the second case $f_{0}$ is assumed to be holomorphic in an open neighborhood of $X_{0} \cup K$. When $K=\emptyset$, we can reduce the first case to the second one by proposition 1.3 ; however, this reduction does not really simplify the proof, its only apparent advantage being that we need not assume the existence of a spray on $Z$ over points in $X_{0}$ which turns out to be very convenient in applications. 
In the first case we can obtain the required homotopy $f_{t}: X \rightarrow Z$ satisfying theorem 1.4 by following the proof of theorem 1.5 in [FP2] (sect. 6), except that we replace the approximation and patching results used there by the corresponding results with interpolation on $X_{0}$, given by theorems 2.2 and 5.1 above. The heart of the proof is an induction scheme (sect. 6 in [FP2]) in which the main ingredient is proposition 5.1 from [FP2].

However, the second case (when $f_{0}$ is holomorphic in a neighborhood of $X_{0} \cup K$ ) requires some modifications because we patch pairs of holomorphic sections on so-called Cartan pairs $(A, B)$ in $X$ where the set $A$ is unbounded (it contains $X_{0} \cup K$ ). We do this by replacing theorem 5.1 by theorem 5.2 above. In this case the globalization process requires small modifications, and we feel that it would be dishonest to leave this entirely to the reader. So we shall stir a middle course by indicating the essential steps of the argument in both cases and referring to [FP2] for the details.

First we must recall from sect. 3 in [FP2] the notion of holomorphic (and continuous) complexes and prisms associated to a given open covering $\mathcal{U}=\left\{U_{j}\right\}$ of the base manifold $X$. Given such a covering $\mathcal{U}$, we denote by $\mathcal{K}(\mathcal{U})$ its nerve (an infinite combinatorial simplicial complex) and by $K(\mathcal{U})$ its geometric realization (an infinite polytope).

Let $h: Z \rightarrow X$ be a given submersion. A holomorphic $\mathcal{K}(\mathcal{U})$-complex with values in $Z$ is a family $f_{*}=\left\{f_{t}: t \in K(\mathcal{U})\right\}$ of holomorphic sections of $Z$, depending continuously on the parameter $t \in K(\mathcal{U})$, where the domain of the section $f_{t} \in f_{*}$ is determined as follows: If $t$ belongs to the $k$-dimensional simplex in $K(\mathcal{U})$ which is determined by $k+1$ open sets $U_{j_{0}}, U_{j_{1}}, \ldots, U_{j_{k}} \in \mathcal{U}$, then $f_{t}$ is a holomorphic section of $Z$ over the set $\cap_{i=0}^{k} U_{j_{i}}$. We also have natural restriction conditions for sections in the family $f_{*}$ on boundaries of simplices in $K(\mathcal{U})$ (see [FP2]). Thus the vertices of $K(\mathcal{U})$ correspond to holomorphic sections on the sets $U_{j} \in \mathcal{U}$, the edges correspond to one-parameter homotopies of sections defined on the intersections $U_{i} \cap U_{j}$, etc. A global section $f: X \rightarrow Z$ may be considered as a 'constant complex', meaning that any section in the associated complex $f_{*}$ is the restriction of $f$ to the appropriate open set in $X$. A holomorphic $k$-prism is a homotopy of holomorphic complexes $f_{*, s}$ depending on a parameter $s \in[0,1]^{k}$. Similarly one defines continuous complexes and prisms with values in $Z$ as collections of continuous sections. We also work with coverings $\mathcal{A}=\left\{A_{i}\right\}$ of $X$ consisting of compact sets; a $\mathcal{K}(\mathcal{A})$-complex is represented by $\mathcal{K}(\mathcal{U})$-complexes for open coverings $\mathcal{U}=\left\{U_{i}\right\}$ of $X$ with $U_{i} \supset A_{i}$ for each $i$, and we identify two complexes whose sections agree near the corresponding sets in $\mathcal{A}$. For details we refer to sect. 3 in [FP2].

Assume for simplicity that $P$ is a singleton; the proof in the general case follows the same pattern. Consider first the case when the restriction $\left.f_{0}\right|_{X_{0}}$ is holomorphic on $X_{0}$ and $f_{0}$ is holomorphic over a neighborhood $U \supset K$ and of a compact $\mathcal{H}(X)$-convex subset $K \subset X$. To harmonize the notation with [FP2] we write $f_{0}=a$. Our goal is to move $a$ by a homotopy which is fixed on $X_{0}$ to a holomorphic section $f: X \rightarrow Z$.

By theorem 4.6 in [FP2] (which follows from the results in sect. 2 of [HL2]) there exists a sequence $\mathcal{A}=\left(A_{0}, A_{1}, A_{2}, \ldots\right)$ of compact, $\mathcal{H}(X)$-convex subsets in $X$, satisfying

(i) $K \subset A_{0} \subset U$ and $X=\cup_{j=0}^{\infty} A_{j}$,

(ii) for each $n \in \mathbf{Z}_{+}$the pair of sets $\left(A^{n}, A_{n+1}\right)$, where $A^{n}=A_{0} \cup A_{1} \cup \cdots \cup A_{n}$, is a $X_{0}$-regular Cartan pair in the sense of def. 4 above, and 
(iii) the submersion $h: Z \rightarrow X$ admits a spray over an open neighborhood of $A_{j}$ for each $j \geq 1$ (but we don't need a spray over $A_{0}$ ).

As in [FP2] we call any finite sequence satisfying (ii) and (iii) a Cartan string, and the entire collection $\mathcal{A}$ is a Cartan covering of $X$. The order of sets in a Cartan string is important because $A^{n} \cap A_{n+1}$ must be Runge in $A_{n+1}$. In [FP2] there was no subvariety $X_{0}$, but the construction in [HL2] shows that we can easily satisfy the $X_{0}$-regularity condition in (ii) by a suitable choice of the set $A_{n}$ at each step.

The sets $A_{j}$ for $j \geq 1$ may be chosen arbitrarily small, subordinate to any open covering of $X$. By proposition 4.7 in [FP2] we can deform the initial section $a$ (which we now consider as a constant $\mathcal{K}(\mathcal{A})$-complex $a_{*, 0}$ ) by a homotopy of continuous $\mathcal{K}(\mathcal{A})$ complexes $a_{*, s}(s \in[0,1])$ into a holomorphic $\mathcal{K}(\mathcal{A})$-complex $a_{*, 1}$. The only additional requirement here is that all sections belonging to any of the complexes $a_{*, s}(s \in[0,1])$ must agree with the initial section $a$ on the intersection of their domains with the subvariety $X_{0}$. This is easily satisfied by first choosing local holomorphic extensions of $\left.a\right|_{X_{0}}$; the homotopies between them are obtained by taking their convex linear combinations with respect to a local linear structure on the fibers of $Z$ (see [FP2]), and hence all sections will agree with $a$ on $X_{0}$.

Applying proposition 5.1 in [FP2] to the holomorphic complex $a_{*, 1}$ we inductively construct a sequence of holomorphic $\mathcal{K}(\mathcal{A})$-complexes $a_{*, n}(n=1,2,3, \ldots)$ which converges as $n \rightarrow \infty$ to a holomorphic section $a_{*, \infty}=f: X \rightarrow Z$. The basic tools that we use in proposition 5.1 from [FP2] in the present case are theorems 2.2 and 5.1; these replace theorems 4.2 and 5.5 from [FP1] that had been used in [FP2]. Here we perform all steps such that all sections and homotopies are fixed on $X_{0}$.

The construction in proposition 5.1 [FP2] is such that the complex $a_{*, n}$ obtained after the first $n$ steps is already constant over the set $A^{n}=\cup_{j=0}^{n} A_{j}$, i.e., it determines a holomorphic section in a neighborhood of $A^{n}$, and the convergence of these sections to $f$ is uniform on compacts in $X$ as $n \rightarrow \infty$. The modification process also gives at each step a homotopy (a 1-prism) between the two adjacent complexes $a_{*, n}$ and $a_{*, n+1}$. This gives in the end a 1-prism connecting the initial section $a=a_{*, 0}$ and the limit section $f$.

Finally, applying the version of proposition 5.1 in [FP2] for continuous prisms, we can homotopically deform the above 1-prism from $a$ to $f$ (keepings the end sections $a$ and $f$ fixed) to another 1-prism which consists of sections over $X$, thereby obtaining a homotopy of sections connecting $a$ to $f$ and satisfying theorem 1.4. Complete details of this argument can be found in sect. 5 and 6 of [FP2]. The same proof applies to $P$-sections. This proves the first part of theorem 1.4 .

Assume now that the initial section $a=f_{0}: X \rightarrow Z$ is holomorphic in an open set $U_{0} \supset X_{0} \cup K$. Our goal is to construct a holomorphic section $f: X \rightarrow Z$ which matches $a$ to a prescribed order $k$ along $X_{0}$ and is obtained from $a$ by a homotopy $f_{t}$ : $X \rightarrow Z$ which is holomorphic near $X_{0}$ and matches $a$ to order $k$ along $X_{0}$. We have two possibilities: either we replace lemma 3.2 with its analogue for functions that vanish to order $k$ along $X_{0}$ (see the remark following the proof of lemma 3.2), or we apply theorem 5.2 in place of theorem 5.1 (and we don't do any gluing along $X_{0}$ ). The first approach works exactly as before. We now elaborate the second approach which has the advantage that no spray is needed 
on $Z$ over neighborhoods of points $x \in X_{0}$. The main step is the following proposition which is similar to proposition 8.2 in [FP2].

6.1 Proposition. Assume that the submersion $h: Z \rightarrow X$ admits a spray over an open neighborhood of each point $x \in X \backslash\left(X_{0} \cup K\right)$. Let $f_{0}: X \rightarrow Z$ be a continuous section which is holomorphic in an open set $U_{0} \supset X_{0} \cup K$. For each compact $\mathcal{H}(X)$-convex subset $L \subset X$ containing $K$ there are an open set $U_{0}^{\prime} \supset X_{0} \cup K$ and a homotopy of continuous sections $f_{t}: X \rightarrow Z(t \in[0,1])$, with $f_{0}$ being the given section, such that for each $t \in[0,1]$, $f_{t}$ is holomorphic in $U_{0}^{\prime}$, it agrees with $f_{0}$ to order $k$ at $X_{0}, f_{t}$ approximates $f_{0}$ uniformly on $K$, and the section $f_{1}$ is holomorphic in an open set $W_{0} \supset X_{0} \cup L$.

Granted proposition 6.1, we complete the proof of theorem 1.4 by exhausting $X$ by a sequence of compact, $\mathcal{H}(X)$-convex subsets $K=L_{0} \subset L_{1} \subset L_{2} \subset \ldots$ and applying proposition 6.1 inductively.

Proof of proposition 6.1. Let $\left(A_{0}, A_{1}, \ldots, A_{n}\right)$ be a Cartan string in $X$ for the pair of sets $K \subset L$, provided by lemma 8.4 in [FP2], satisfying

(i) $K \cup\left(X_{0} \cap L\right) \subset A_{0} \subset U_{0}$;

(ii) for $j=1,2, \ldots, n$ we have $A_{j} \cap\left(X_{0} \cup K\right)=\emptyset$ and $A_{j} \subset U_{k}$ for some $k=k(j) \geq 1$;

(iii) $L=\cup_{0 \leq j \leq n} A_{j}$.

By (ii) we have $A_{j} \cap X_{0}=\emptyset$ for all $j \geq 1$ and hence this string is $X_{0}$-regular. We now inductively apply proposition 5.1 in [FP2], making certain to satisfy the interpolation condition along $X_{0}$. In the process we must patch holomorphic sections on Cartan pairs $\left(A^{j}, A_{j+1}\right)$, where $A^{j}=A_{0} \cup A_{2} \cup \ldots \cup A_{j}$, as well as on some other Cartan pairs which do not intersect $X_{0}$. While no change in the procedure is needed for the second case, the first case must be modified in such a way that the resulting sections will be holomorphic in a neighborhood of $X_{0}$ and will interpolate the initial section. Set $A_{0}^{\prime}=A_{0} \cup X_{0}$ and $\mathcal{A}^{\prime}=\left(A_{0}^{\prime}, A_{1}, \ldots, A_{n}\right)$. For each $j$ the set $A^{j}=\cup_{l=0}^{j} A_{l}$ is $\mathcal{H}(X)$-convex, and hence the same is true for $\left(A^{\prime}\right)^{j}=X_{0} \cup A^{j}=A_{0}^{\prime} \cup A_{1} \cup \ldots \cup A_{j}$. Thus $\mathcal{A}^{\prime}$ satisfies all properties of an $X_{0}$-regular Cartan string, except that the initial set $A_{0}^{\prime}$ is unbounded.

By proposition 4.7 in [FP2] we can associate to $f_{0}$ the initial holomorphic $\mathcal{K}\left(\mathcal{A}^{\prime}\right)$ complex $f_{*, 0}$ such that the section associated to $A_{0}^{\prime}$ equals the restriction of $f_{0}$ to a suitable open neighborhood of $A_{0}^{\prime}$ in $X$. We now modify this complex $f_{*, 0}$ by inductively applying proposition 5.1 in [FP2], except that we perform the gluing of sections on Cartan pairs $\left(\left(A^{\prime}\right)^{j}, A_{j+1}\right)$ by appealing to theorem 5.2 instead of theorem 5.1. The neighborhood of $A_{0}^{\prime}$ on which the new section (the result of the gluing) is defined may shrink at each step without any detrimental consequences. After $n$ steps we obtain a holomorphic section $f_{1}$ in an open set $W_{0}^{\prime} \supset X_{0} \cup L$ which can be connected to $f_{0}$ by a holomorphic homotopy $f_{t}: W_{0}^{\prime} \rightarrow Z(t \in[0,1])$.

It remains to extend $f_{t}$ to $X$ by modifying it outside a smaller neighborhood of $X_{0} \cup L$. This is done as usual by taking $f_{t \chi(x)}(x)(x \in X, t \in[0,1])$, where $\chi: X \rightarrow[0,1]$ is a smooth cut-off function which vanishes outside $W_{0}^{\prime}$ and equals one in a smaller open set $W_{0} \supset X_{0} \cup L$. This completes the proof. 


\section{\&7. Submersions with stratified sprays.}

We can extend theorem 1.4 to submersions with stratified sprays over Stein spaces (compare with sec. 3.1 in Gromov [Gro]), a situation which arises naturally in global analytic geometry. An important special case was considered in 1966 by Forster and Ramspott [FR].

Let $X$ and $Z$ be complex spaces, possibly with singularities. A holomorphic map $h: Z \rightarrow X$ is said to be a submersion (of co-rank $k$ ) if it is locally near each point $z_{0} \in Z$ equivalent (by a fiber preserving biholomorphic map) to a projection $p: U \times V \rightarrow U$, where $U \subset X$ is an open set in $X$ containing $x_{0}=h\left(z_{0}\right)$ and $V$ is an open set in some $\mathbf{C}^{d}$.

Assume now that the base $X$ of a submersion $h: Z \rightarrow X$ is a Stein space which is stratified by a descending chain of closed complex subspaces $X=X_{m} \supset X_{m-1} \supset \cdots \supset$ $X_{1} \supset X_{0}$ such that each stratum $Y_{k}=X_{k} \backslash X_{k-1}(1 \leq k \leq m)$ is regular and the restricted submersion $h:\left.Z\right|_{Y_{k}} \rightarrow Y_{k}$ admits a spray over a small neighborhood of any point $x \in Y_{k}$ (def. 2 in sec. 1). Then the following holds:

Any continuous section $f_{0}: X \rightarrow Z$ such that $\left.f_{0}\right|_{X_{0}}$ is holomorphic can be deformed to a holomorphic section $f_{1}: X \rightarrow Z$ by a homotopy $f_{t}: X \rightarrow Z(t \in[0,1])$ that is fixed on $X_{0}$.

A similar result holds for $P$-sections whose restriction to $X_{0}$ is holomorphic (see def. 3 and theorem 1.4).

One can prove this by induction over the strata $X_{k}$ as follows. Suppose that for some $k \geq 1$ we have already constructed a continuous section $f_{k-1}: X \rightarrow Z$ that is holomorphic over $X_{k-1}$ and satisfies $f_{k-1}=f_{0}$ on $X_{0}$. We first apply proposition 1.3 (which holds also for complex spaces) to make $f_{k-1}$ holomorphic in an open neighborhood of $X_{k-1}$ in $X$ and continuous elsewhere. We then apply theorem 1.4 , with $\left(X, X_{0}\right)$ replaced by the pair $\left(X_{k}, X_{k-1}\right)$, to deform $f_{k-1}$ through a homotopy to a continuous section $f_{k}: X \rightarrow Z$ that is holomorphic over $X_{k}$ and matches $f_{0}$ on $X_{0}$. In finitely many steps we obtain a holomorphic section over $X$.

The construction $f_{k}$ requires a version of theorem 1.4 in which $X$ is a Stein space whose singular locus is contained in the subvariety $X_{0}$ where the initial section is already holomorphic. Such a result can be proved by small changes in the proof of proposition 4.2 and theorem 5.2 as follows. Each Stein space $X$ of dimension $n$ admits a holomorphic map $g: X \rightarrow \mathbf{C}^{2 n+1}$ which is a homeomorphism of $X$ onto a closed Stein subspace $\tilde{X}=$ $g(X) \subset \mathbf{C}^{2 n+1}$ and is an injective immersion on the regular part of $X[\mathrm{GuR}]$. Observe that the attaching of pairs of holomorphic sections (proposition 4.2 and theorem 5.2) needs to be carried out only on Cartan pairs $(A, B)$ in $X$ for which the set $C=A \cap B$ is contained in the regular part of $X$. Since $g$ is regular and injective there, we may transfer the data for the patching problem (the transition map $\psi$ in proposition 4.2 and theorem 5.2) to a suitably chosen open neighborhood of $g(C)$ in $\mathbf{C}^{2 n+1}$. The solution obtained on a Cartan pair in $\mathbf{C}^{2 n+1}$ is then pulled back to $X$ by $g$. The globalization scheme in sect. 6 goes through without changes. We leave out further details.

Remark. It seems that the first examples of 'submersion with stratified sprays' satisfying the h-principle were the Endromisbündel of Forster and Ramspott [FR]. These are submersions $Z \rightarrow X$, where $Z$ is an open subset in $X \times M(s, r)(M(r, s)=$ the set of complex $s \times r$ 
matrices) and the restrictions $\left.Z\right|_{Y_{k}} \rightarrow Y_{k}$ to certain strata $Y_{k}=X_{k} \backslash X_{k-1}$ are holomorphic fiber bundles with complex Grassman manifolds as fibers. The corresponding h-principle, proved in $[\mathrm{FR}]$, had many interesting applications, e.g. to obtain the minimal number of global generators of a given coherent analytic sheaf over $X$.

Acknowledgements. We wish to thank Bo Berndtsson for his help in the proof of lemma 3.1. The first author acknowledges partial support by the NSF, by the Vilas foundation at the University of Wisconsin-Madison and by the Ministry of Science of the Republic of Slovenia. The second author was supported in part by the Ministries of Science and of Education in Slovenia. A part of the work was done while the second author was visiting the Mathematics Department at the University of Wisconsin-Madison in the Spring of 2000 and she wishes to thank this institution for its hospitality.

\section{References.}

[AAC] K. Adachi, M. Andersson, H.R. Cho: $L^{p}$ and $H^{p}$ extensions of holomorphic functions from subvarieties of analytic polyhedra. Pacific J. Math. 189 (1999), 201-210.

[Car] H. Cartan: Espaces fibrés analytiques. Symposium Internat. de topologia algebraica, Mexico, 97-121 (1958). (Also in Oeuvres, vol. 2, Springer, New York, 1979.)

[Dem] J.-P. Demailly: Un exemple de fibré holomorphe non de Stein à fibre $\mathbf{C}^{\mathbf{2}}$ ayant pour base le disque ou le plan. Invent. Math. 48, 293-302 (1978).

[FR] O. Forster and K. J. Ramspott: Analytische Modulgarben und Endromisbündel. Invent. Math. 2, 145-170 (1966).

[FP1] F. Forstnerič and J. Prezelj: Oka's principle for holomorphic fiber bundles with sprays. Math. Ann. 317 (2000), 117-154.

[FP2] F. Forstnerič and J. Prezelj: Oka's principle for holomorphic submersions with sprays. Preprint, 1999.

[Gr1] H. Grauert: Approximationssätze für holomorphe Funktionen mit Werten in komplexen Räumen. Math. Ann. 133, 139-159 (1957).

[Gr2] H. Grauert: Holomorphe Funktionen mit Werten in komplexen Lieschen Gruppen. Math. Ann. 133, 450-472 (1957).

[Gr3] H. Grauert: Analytische Faserungen ber holomorph-vollstndigen Rumen. Math. Ann. 135, 263-273 (1958).

[GRe] H. Grauert, R. Remmert: Theory of Stein Spaces. Grundl. Math. Wiss. 227, Springer, New York, 1977.

[Gro] M. Gromov: Oka's principle for holomorphic sections of elliptic bundles. J. Amer. Math. Soc. 2, 851-897 (1989).

[GuR] C. Gunning, H. Rossi: Analytic functions of several complex variables. PrenticeHall, Englewood Cliffs, 1965.

[Hen] G. M. Henkin: Continuation of bounded holomorphic functions from submanifolds in general position in a strictly pseudoconvex domain. (Russian) Izv. Akad. Nauk SSSR Ser. Mat. 36 (1972), 540-567. 
[HL1] G. M. Henkin, J. Leiterer: Theory of functions on complex manifolds. AkademieVerlag, Berlin, 1984.

[HL2] G. Henkin, J. Leiterer: The Oka-Grauert principle without induction over the basis dimension. Math. Ann. 311, 71-93 (1998).

[Hör] L. Hörmander: An Introduction to Complex Analysis in Several Variables, 3rd ed. North Holland, Amsterdam, 1990.

[Pre] J. Prezelj: Interpolation of Embeddings of Stein Manifolds on Discrete Sets. Preprint, 2000.

[RRu] J.-P. Rosay, W. Rudin: Holomorphic maps from $\mathbf{C}^{n}$ to $\mathbf{C}^{n}$. Trans. Amer. Math. Soc. 310, 47-86 (1988)

[Siu] Y.T. Siu: Every Stein subvariety admits a Stein neighborhood. Invent. Math. 38, 89-100 (1976).

Franc Forstnerič

Department of Mathematics

University of Wisconsin

Madison, WI 53706, USA

Current address:

IMFM, University of Ljubljana

Jadranska 19

1000 Ljubljana, Slovenia
Jasna Prezelj

IMFM, University of Ljubljana

Jadranska 19

1000 Ljubljana, Slovenia 